\title{
Perturbation results involving the 1-Laplace operator
}

\author{
Samuel Littig Friedemann Schuricht*
}

August 30, 2018

We consider perturbed eigenvalue problems of the 1-Laplace operator and verify the existence of a sequence of solutions. It is shown that the eigenvalues of the perturbed problem converge to the corresponding eigenvalue of the unperturbed problem as the perturbation becomes small. The results rely on nonsmooth critical point theory based on the weak slope.

Keywords: 1-Laplace operator, eigenvalue problems, perturbation, nonsmooth critical point theory, weak slope

\section{Introduction}

Investigations of perturbations of the eigenvalue problem of the $p$-Laplace operator

$$
-\Delta_{p} u+f(x, u)=\lambda|u|^{p-2} u \quad \text { on } \Omega, \quad u=0 \quad \text { on } \partial \Omega
$$

where

$$
\Delta_{p} u:=\operatorname{div}|D u|^{p-2} D u \quad(1<p<\infty),
$$

gained a lot of interest in the past. A weak solution $u \in W_{0}^{1, p}(\Omega) \backslash\{0\}$ is called eigenfunction, the scalar $\lambda$ eigenvalue, and the tuple $(\lambda, u)$ eigensolution of equation (1.1). The function $f$ is considered as perturbation and one typically assumes that $f$ is small provided $u$ is small, such that $(\lambda, 0)$ is a trivial eigensolution of (1.1) for any $\lambda>0$. For $f=0$ we have the (unperturbed) eigenvalue problem of the $p$-Laplace operator.

*Both authors supported by DFG project "Variational problems related to the 1-Laplace operator". 
It is well known that there exists an unbounded sequence of eigenvalues

$$
0<\lambda_{1, p}<\lambda_{2, p} \leq \lambda_{3, p} \leq \ldots
$$

of the unperturbed $p$-Laplace operator with corresponding eigenfunctions $u_{k, p}$. Clearly, any multiple of $u_{k, p}$ is also eigenfunction for $\lambda_{k, p}$. Thus the $\left(\lambda_{k, p}, 0\right)$ are bifurcation points on the branch of trivial solutions $(\lambda, 0)_{\lambda \in \mathbb{R}}$ of the unperturbed problem (1.1) and a natural question is how far this situation is preserved under small perturbations.

Under suitable assumptions on $f$, the operator $Q: W_{0}^{1, p}(\Omega) \rightarrow W_{0}^{1, p}(\Omega)$ with

$$
Q(u):=\left(-\Delta_{p}\right)^{-1}\left(\lambda|u|^{p-2} u-f(x, u)\right)
$$

turns out to be compact and small as $u$ is small. Hence we may calculate the Leray-Schauder mapping degree of $u \mapsto u-Q(u)$. If the eigenvalue $\lambda_{k, p}$ is simple (which is always the case for $\lambda_{1, p}$ when $\Omega$ is connected), there exists a continuous curve $\left(\lambda_{t}, u_{t}\right)_{t \in \mathbb{R}}$ of eigensolutions of the perturbed problem (1.1) crossing the branch of trivial solutions at $\left(\lambda_{k, p}, 0\right)$ (cf. del Pino \& Manásevich [9] and the survey notes of Peral [19]). Consequently, if $\lambda_{k, p}$ is simple, $\left(\lambda_{k, p}, 0\right)$ is a bifurcation point of the perturbed problem as well and the eigenvalue $\lambda_{k, p}$ of the $p$-Laplace operator turns out to be a bifurcation value of the perturbed $p$-Laplace eigenvalue problem (1.1).

A key point in the investiagtion of (1.1) is the underlying variational structure. In fact the unperturbed problem (1.1) (i.e. $f=0$ ) is the Euler-Lagrange equation of the variational problem

$$
\mathcal{E}_{p}(v):=\frac{1}{p} \int_{\Omega}|D v|^{p} \mathrm{~d} x \rightarrow \underset{v \in W_{0}^{1, p}(\Omega)}{\operatorname{Min} !}
$$

subject to

$$
\mathcal{G}_{p}(v):=\frac{1}{p} \int_{\Omega}|v|^{p} \mathrm{~d} x=1
$$

In other words, any critical point $u$ of (1.3), (1.4) turns out to be an eigenfunction of the $p$-Laplace operator for the eigenvalue $\lambda=p \mathcal{E}_{p}(u)$ (which equals the Lagrange multiplier of the constrained variational problem). Moreover each eigenfunction of the unperturbed equation (1.1) is a multiple of a critical point of (1.3), (1.4).

Notice that an unbounded sequence of eigenvalues $\lambda_{k, p}$ of the $p$-Laplace operator as mentioned in (1.2) can be obtained by minimax methods within Ljuster- 
nik-Schnirelman theory where one has

$$
\lambda_{k, p}=\inf _{S \in \mathscr{S}^{k, p}} \sup _{v \in S} \mathcal{E}_{p}(v) .
$$

Here the $\mathscr{S}^{k, p}$ are suitable classes of subsets of $W_{0}^{1, p}(\Omega)$ expressing some topological property of the level sets of $\mathcal{E}_{p}$ by means of some topological index $k$. It is well known that these eigenvalues are continuous in $p$ on $[1, \infty)$ (cf. Parini [18], Littig \& Schuricht [14]). For $p>1$ the eigenvalue problem is studied in wide detail with contributions of many authors. Let us just mention Garcia Azorero \& Peral Alonso [12], who seem to have studied the problem first, and the long list of references contained in Peral [19].

When studying (1.1), one usually distinguishes three cases depending on the growth of $f$. Here a typical assumption on $f$ is

$$
|f(x, u)| \leq C\left(1+|u|^{r-1}\right) .
$$

For $1 \leq r<p^{*}:=\frac{n p}{n-p}$ the problem is called subcritical, for $r=p^{*}$ it is called critical, and for $r>p^{*}$ it is called supercritical. If $p \geq n$ the problem is always subcritical. Usually the subcritical case is the most easy one to treat. In the critical case we may expect similar results as in the subcritical case, but the techniques for the proofs are more involved. In the supercritical case nonexistence of solutions may occur (cf. [19]).

The intention of the present paper is to study such bifurcation problems for the degenerate limit case $p=1$. Taking into account two types of perturbations, we cover problems that are formally given by

$$
-\operatorname{div} \frac{D u}{|D u|}+f(x, u)=\lambda \frac{u}{|u|} \quad \text { on } \Omega, \quad u=0 \quad \text { on } \partial \Omega,
$$

and by

$$
-\operatorname{div} \frac{D u}{|D u|}=\lambda\left(\frac{u}{|u|}+g(x, u)\right) \quad \text { on } \Omega, \quad u=0 \quad \text { on } \partial \Omega
$$

Notice that already the (unperturbed) eigenvalue problem of the 1-Laplace operator (i.e. $f=0$ or $g=0$ ) is highly degenerated, since the equations above are not well defined at points where $u(x)=0$ or $D u(x)=0$. Having in mind that typically the first eigenfunction of the 1-Laplace operator is a multiple of a characteristic function vanishing on a set of positive measure, it becomes clear that the equations need some careful justification. Instead of $W_{0}^{1,1}(\Omega)$ one has to work in $B V(\Omega)$ and the homogeneous boundary conditions have to be considered 
in a more general sense than the usual trace in $B V(\Omega)$. Then it turns out that the unperturbed problem is related to the variational problem

$$
\mathcal{E}_{T V}(v):=\int_{\Omega} \mathrm{d}|D v|+\int_{\partial \Omega}|v| \mathrm{d} \mathcal{H}^{n-1} \rightarrow \underset{v \in B V(\Omega)}{\operatorname{Min} !}
$$

subject to

$$
\mathcal{G}_{1}(v)=\int_{\Omega}|v| \mathrm{d} x=1
$$

(cf. Kawohl \& Schuricht [13]). With methods from convex analysis and nonsmooth critical point theory one can show that critical points of problem (1.8), (1.9) (in the sense of weak slope) satisfy the Euler-Lagrange equation

$$
-\operatorname{div} z=\lambda s \quad \text { on } \Omega \text {. }
$$

Here $z \in L^{\infty}(\Omega)$ is some vector field giving sense to $\frac{D u}{|D u|}$ and $s: \Omega \rightarrow[-1,1]$ is some sign function giving sense to $\frac{u}{|u|}$ (cf. [13]). Existence of a sequence of eigenfunctions $\left(u_{k, 1}\right)_{k \in \mathbb{N}}$ of the 1-Laplace operator with an unbounded sequence of corresponding eigenvalues

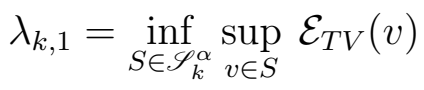

was verified in Milbers \& Schuricht [16] by minimax methods. While in [16] the classes $\mathscr{S}_{k}^{\alpha}$ are defined by means of category as topological index, we know from Littig \& Schuricht [14] that these eigenvalues $\lambda_{k, 1}$ coincide with that using

$$
\mathscr{S}_{k}^{\alpha}:=\left\{S \subseteq L^{1}(\Omega) \text { compact, symmetric ; } \mathcal{G}_{1}=1 \text { on } S, \text { gen }_{L^{1}} S \geq k\right\}
$$

with genus gen $L_{L^{1}} S$ as topological index in (1.11).

Investigating bifurcation for the formal problems (1.6) and (1.7) we are confronted with the question how to define solutions. We have to realize that even in the unperturbed case the well-defined interpretation (1.10) of the formal equation has too many solutions and cannot identify reasonable solutions of the problem (cf. Kawohl \& Schuricht [13], Milbers \& Schuricht [17]). Therefore we have to define solutions of (1.6) and (1.7) as critical points (in the sense of weak slope) of a related variational problem. In this sense we first verify the existence of a sequence of eigensolutions $\left(\lambda_{k, \alpha}, u_{k, \alpha}\right)_{k \in \mathbb{N}}$ with critical values $c_{k, \alpha}$ for a class of problems covering (1.6) and a sequence of eigensolutions $\left(\lambda_{k, \beta}, u_{k, \beta}\right)_{k \in \mathbb{N}}$ with critical values $c_{k, \beta}$ for a class of problems covering (1.7) for each sufficiently small parameter $\alpha>0$ and $\beta>0$, respectively. In both cases we assume that the perturbation is of subcritical type, i.e. $1<p<\frac{n}{n-1}$. The parameters $\alpha$ 
and $\beta$ correspond to the norm of the eigenfunctions and, thus, they reflect the magnitude of the perturbation. The perturbation is shown to vanish as $\alpha$ or $\beta$ tend to zero provided we have the stronger condition $1<p<\frac{n+1}{n}$.

Finally we prove

$$
\lambda_{k, 1}=\lim _{\alpha \rightarrow 0} \lambda_{k, \alpha} \quad \text { and } \quad \lambda_{k, 1}=\lim _{\beta \rightarrow 0} \lambda_{k, \beta}
$$

for any $k \in \mathbb{N}$. Since all points $(\lambda, 0)_{\lambda \in \mathbb{R}}$ may be considered as trivial solution of the perturbed eigenvalue problem, (1.12) shows that the minimax eigenvalues $\lambda_{k, 1}$ of the (unperturbed) 1-Laplace operator according to (1.11) are bifurcation values for the perturbed eigenvalue problems (1.6) and (1.7).

Let us mention that Degiovanni \& Magrone [6] treated the critical case (which is not covered by our results). They proved existence of nontrivial solutions of (1.6) for any $\lambda>\lambda_{k, 1}$ and the perturbation $f(x, u)=-|u|^{\frac{n}{n-1}-2} u$. Here a different method is used that relies on truncation techniques of $B V$-functions and exploits the specific form of the perturbation.

In Section 2 we precisely formulate the two types of perturbed eigenvalue problems and justify related quantities. The main results are stated in Section 3. As preparation for the proofs, Section 4 presents tools from nonsmooth critical point theory and some general norm estimates. In Section 5 we give the proofs of the main results.

Notation and Conventions: By $L^{p}(\Omega)$ we denote the usual Lebesgue space of $p$-integrable functions with norm $\|\cdot\|_{p}$ and by $W_{0}^{1, p}(\Omega)$ the Sobolev space of $p$-integrable functions having $p$-integrable weak derivatives and zero trace. $C_{\mathrm{BV}}$ is the embedding constant of $W_{0}^{1,1}(\Omega)$ (with norm $\|D v\|_{1}$ ) in $L^{\frac{n}{n-1}}(\Omega)$, i.e. it is the optimal constant in

$$
\|v\|_{\frac{n}{n-1}} \leq C_{\mathrm{BV}}\|D v\|_{1} \quad \text { for all } v \in W_{0}^{1,1}(\Omega) .
$$

$B V(\Omega)$ stands for the space of functions of bounded variation. With the usual convention of identifying $v \in L^{1}(\Omega)$ with its extension by zero on $\mathbb{R}^{n} \backslash \Omega$, we have for all $v \in B V(\Omega)$

$$
\mathcal{E}_{T V}(v)=\int_{\mathbb{R}^{n}} \mathrm{~d}|D v|=\int_{\Omega} \mathrm{d}|D v|+\int_{\partial \Omega}|v| \mathrm{d} \mathcal{H}^{n-1}
$$

(cf. [10]). Due to Theorem 3.1 in [14] and the Poincaré inequality, $\mathcal{E}_{T V}$ is a norm on $B V(\Omega)$ equivalent to the standard norm. $C_{c}^{\infty}(\Omega)$ is the space of test functions having compact support.

We write $\lambda_{k, p}(p>1)$ for the variational eigenvalues of the $p$-Laplace operator as given in (1.5) and $\lambda_{k, 1}$ always stands for the eigenvalues of the (unperturbed) 
1-Laplace operator according to (1.11). Without danger of confusion we use $\lambda_{k, \alpha}$ and $\lambda_{k, \beta}$ for the eigenvalues of the perturbed 1-Laplace operator for perturbations of the type given in Section 3.1 and Section 3.2, respectively. Analogously we denote the corresponding eigenfunctions and critical values.

The set-valued sign function Sgn on $\mathbb{R}$ is

$$
\operatorname{Sgn}(s):=\left\{\begin{array}{cc}
\{1\} & \text { if } s>0 \\
{[-1,1]} & \text { if } s=0 \\
\{-1\} & \text { if } s<0
\end{array}\right.
$$

and $\mathbb{S}^{k-1}$ denotes the $(k-1)$-dimensional unit sphere in $\mathbb{R}^{k}$.

For a Banach space $X$ and its dual $X^{*}$, the duality pairing is given by $\langle\cdot, \cdot\rangle$. By $B_{\rho}(u)$ we denote the open $\rho$-ball around $u$, by $B_{\rho}(M)$ the open $\rho$-neighborhood of the set $M$, by $\bar{M}$ the closure of $M$, by $I_{M}$ the indicator function of $M$, and by $\chi_{M}$ the characteristic function of $M$. We write $\operatorname{gen}_{X} S$ for the genus of a symmetric set $S \subseteq X \backslash\{0\}$ (cf. [21, Chap. 44.3] for basic properties). For a scalar function $\mathcal{F}: X \rightarrow \mathbb{R}$ we use $\partial \mathcal{F}(u)$ to denote the convex subdifferential at $u$ for a convex $\mathcal{F}$ and Clarke's generalized gradient at $u$ for a locally Lipschitz continuous $\mathcal{F}$. Clarke's generalized directional derivative of $\mathcal{F}$ at $u$ in direction $v$ is given by $\mathcal{F}^{0}(u ; v)$ (cf. [3]). For a continuous or merely lower semicontinuous $\mathcal{F}: M \rightarrow \mathbb{R}$ on a metric space $M$, the weak slope of $\mathcal{F}$ at $u$, denoted by $|d \mathcal{F}|(u)$, is a nonnegative real number that describes somehow the slope of $\mathcal{F}$ on some neighborhood of $u$ and can be considered as some replacement of $\left\|\mathcal{F}^{\prime}(u)\right\|$ in the smooth case (cf. Section 4.1 for some brief introduction).

\section{Formulation of the problem}

We always assume that $\Omega \subseteq \mathbb{R}^{n}$ is open and bounded with Lipschitz boundary and that $1<p<\infty$. First we study perturbed eigenvalue problems that cover problems formally given by

$$
-\operatorname{div} \frac{D u}{|D u|}+f(x, u)=\lambda \frac{u}{|u|} \quad \text { on } \Omega, \quad u=0 \quad \text { on } \partial \Omega .
$$

More precisely we consider critical points of a constraint variational problem

$$
\mathcal{E}_{T V}(v)+\mathcal{E}_{\mathrm{Per}}(v) \rightarrow \underset{v \in L^{p}(\Omega)}{\operatorname{Min} !}
$$

subject to

$$
\mathcal{G}_{1}(v):=\int_{\Omega}|v| \mathrm{d} x=\alpha
$$


Here $\mathcal{E}_{\mathrm{Per}}: L^{p}(\Omega) \rightarrow \mathbb{R}$ be a suitable locally Lipschitz continuous functional and we identify $\mathcal{E}_{T V}$ with its extension on $L^{p}(\Omega)$ for $1<p<\frac{n}{n-1}$ given by

$$
\mathcal{E}_{T V}(v):= \begin{cases}\int_{\Omega} \mathrm{d}|D v|+\int_{\partial \Omega}|v| \mathrm{d} \mathcal{H}^{n-1} & \text { on } B V(\Omega) \\ \infty & \text { on } L^{p}(\Omega) \backslash B V(\Omega)\end{cases}
$$

Obviously $\mathcal{E}_{T V}$ is convex and it is the the lower semicontinuous extension of $\int_{\Omega}|D v| \mathrm{d} x$ from $v \in W_{0}^{1,1}(\Omega)$ on $L^{p}(\Omega)$ (cf. [13] and [14]). We call $u \in L^{p}(\Omega)$ critical point of (2.2), (2.3) if $u$ is a critical point with respect to the weak slope of

$$
\mathcal{E}=\mathcal{E}_{T V}+\mathcal{E}_{\text {Per }}
$$

in the metric space

$$
K_{\alpha}:=\left\{v \in L^{p}(\Omega) ; \mathcal{G}_{1}(v)=\alpha\right\}
$$

i.e. if $|d \mathcal{E}|(u)=0$ (cf. Section 4.1 and Degiovanni \& Marzocchi [7]). This is equivalent to $u$ being a critical point of

$$
\tilde{\mathcal{E}}=\mathcal{E}_{T V}+\mathcal{E}_{\mathrm{Per}}+I_{\left\{\mathcal{G}_{1}=\alpha\right\}}
$$

on the metric space $L^{p}(\Omega)$, i.e. $|d \tilde{\mathcal{E}}|=0$, where

$$
I_{\left\{\mathcal{G}_{1}=\alpha\right\}}(v)= \begin{cases}0 & \text { when } \mathcal{G}_{1}(v)=\alpha \\ \infty & \text { otherwise }\end{cases}
$$

is the indicator function of $K_{\alpha}$ (cf. also Milbers \& Schuricht [15]).

With this definition at hand we can apply a nonsmooth version of LjusternikSchnirelman theory to get a sequence $\left(u_{k, \alpha}\right)_{k \in \mathbb{N}}$ of eigenfunctions of the perturbed problem (2.2), (2.3) for each parameter $\alpha>0$. As necessary condition each eigensolution $(\lambda, u)$ satisfies an Euler-Lagrange equation of the type

$$
-\operatorname{div} z+u^{*}=\lambda s
$$

where $z$ and $s$ are related to $u$ as in the unperturbed case (cf. (1.10) ) and $u^{*} \in \partial \mathcal{E}_{\text {Per }}(u)$. Also in the perturbed case the parameter $\lambda \in \mathbb{R}$ will be called eigenvalue of the eigenfunction $u$. Essential ingredients in our analysis will be some Palais-Smale condition (short (PS)-condition), which requires special care, and the so-called epigraph condition (short (epi)-condition) that rules out "artificial" critical points on the epigraph of our merely lower semicontinuous functional and it can be treated rather straightforward. 
As a second type of perturbation we cover problems formally given by

$$
-\operatorname{div} \frac{D u}{|D u|}=\lambda\left(\frac{u}{|u|}+g(x, u)\right) \quad \text { on } \Omega, \quad u=0 \quad \text { on } \partial \Omega .
$$

More precisely we study critical points of constrained variational problems

$$
\mathcal{E}_{T V}(v) \rightarrow \underset{v \in L^{p}(\Omega)}{\operatorname{Min} !}
$$

subject to

$$
\mathcal{G}_{1}(v)+\mathcal{G}_{P e r}(v)=\beta
$$

where $\mathcal{G}_{\text {Per }}: L^{p}(\Omega) \rightarrow \mathbb{R}$ is a suitable locally Lipschitz continuous functional. Here $u \in L^{p}(\Omega)$ is a critical point of (2.7), (2.8) if $u$ is a critical point of $\mathcal{E}_{T V}$ in the metric space

$$
K_{\beta}:=\left\{v \in L^{p}(\Omega) ; \mathcal{G}_{1}(v)+\mathcal{G}_{\mathrm{Per}}(v)=\beta\right\}
$$

i.e. $\left|d \mathcal{E}_{T V}\right|(u)=0$ or, equivalently, if $u$ is a critical point of

$$
\mathcal{E}=\mathcal{E}_{T V}+I_{\left\{\mathcal{G}_{1}+\mathcal{G}_{P e r}=\beta\right\}}
$$

on $L^{p}(\Omega)$, i.e. $|d \mathcal{E}|(u)=0$. This way we again obtain a sequence $\left(u_{k, \beta}\right)_{k \in \mathbb{N}}$ of eigenfunctions of the perturbed problem (2.7), (2.8) for each parameter $\beta>0$ and the eigensolutions satisfy an Euler-Lagrange equation of the form

$$
-\operatorname{div} z=\lambda\left(s+u^{*}\right)
$$

where $z$ and $s$ are related to $u$ as before and $u^{*} \in \partial \mathcal{G}_{P e r}(u)$. Again $u$ will be called eigenfunction and $\lambda$ the corresponding eigenvalue for this type of perturbation. In contrast to the perturbation of the first type, the (PS)-condition is a simple immediate consequence of the compact embedding from $B V(\Omega)$ in $L^{p}(\Omega)$, but the verification of the (epi)-condition turns out to be more delicate.

Remark 2.1. Note that the weak slope and, thus, our definition of criticality depends on the specific choice of the metric. This issue was already addressed in Milbers \& Schuricht [15] and Littig \& Schuricht [14]. It turns out that, for any $p \in\left[1, \frac{n}{n-1}\right)$, the minimax construction as in (1.11) provides eigenfunctions $u_{k, 1}$ of the (unperturbed) 1-Laplace operator that are critical points in $L^{p}(\Omega)$ with respect to the $L^{p}$-metric. However it is not clear whether $L^{p}$-critical points are also $L^{q}$-critical points for $p \neq q$ in general. Alternatively one could consider critical points in $B V(\Omega)$ with respect to the stronger $B V$-norm. In the one- 
dimensional case, for $\Omega=(0,1) \subseteq \mathbb{R}$, it can be shown that this leads to a much larger set of critical points and it seems that, in general, the BV-norm is too strong to get a reasonable set of critical points (cf. Milbers \& Schuricht [17]).

As prototype for perturbations $\mathcal{E}_{\text {Per }}, \mathcal{G}_{\text {Per }}$ we have in mind functionals of the form

$$
\mathcal{E}_{\mathrm{Per}}(v)=\int_{\Omega} \int_{0}^{v(x)} f(x, s) \mathrm{d} s \mathrm{~d} x, \quad \mathcal{G}_{\mathrm{Per}}(v)=\int_{\Omega} \int_{0}^{v(x)} g(x, s) \mathrm{d} s \mathrm{~d} x .
$$

In order to derive general properties for this kind of functionals we use the notation

$$
\mathcal{F}(v):=\int_{\Omega} \int_{0}^{v(x)} f(x, s) \mathrm{d} s \mathrm{~d} x .
$$

For the integrand $f: \Omega \times \mathbb{R} \rightarrow \mathbb{R}$ we assume that

(f1) $f$ is locally integrable on $\Omega \times \mathbb{R}$ and $F: \Omega \times \mathbb{R} \rightarrow \mathbb{R}$ defined by

$$
F(x, t):=\int_{0}^{t} f(x, s) \mathrm{d} s
$$

is a Carathéodory function,

(f2) there is $C_{\text {Per }}>0$ such that

$$
|f(x, s)| \leq p C_{\text {Per }}|s|^{p-1} \quad \text { for all } s \in \mathbb{R} \text { and a.e. } x \in \Omega \text {, }
$$

(f3) $f(x, \cdot)$ is odd for a.e. $x$, i.e.

$$
f(x, s)=-f(x,-s) \text { for all } s \in \mathbb{R} \text { and a.e. } x \in \Omega,
$$

A standard example for $f$ would be

$$
f(x, s)=|s|^{p-2} s .
$$

In the following theorem, which is proved in Section 5.1, we summarize several properties of functional $\mathcal{F}$ given in (2.10) .

Theorem 2.2. Let $\Omega \subseteq \mathbb{R}^{n}$ be open and bounded with Lipschitz boundary and let $f$ satisfy conditions (f1)-(f3) with $p \in(1, \infty)$. Then $\mathcal{F}: L^{p}(\Omega) \rightarrow \mathbb{R}$ according to (2.10) is well defined with

(1) $\mathcal{F}$ is even, i.e. $\mathcal{F}(u)=\mathcal{F}(-u)$ for all $u \in L^{p}(\Omega)$,

(2) $\mathcal{F}(u)=\mathcal{F}(|u|)$ for all $u \in L^{p}(\Omega)$,

(3) $\mathcal{F}$ is Lipschitz continuous on bounded subsets of $L^{p}(\Omega)$ and, thus, locally Lipschitz continuous on $L^{p}(\Omega)$, 
(4) for $u^{*} \in \partial \mathcal{F}(u) \subseteq L^{p^{\prime}}(\Omega)$ one has

$$
\begin{gathered}
\left.u^{*}(x) \in \underset{s \rightarrow u(x)}{\operatorname{ess} \inf } f(x, s), \underset{s \rightarrow u(x)}{\operatorname{ess} \sup _{s \rightarrow}} f(x, s)\right] \quad \text { for a.e. } x \in \Omega, \\
\left\|u^{*}\right\|_{p^{\prime}} \leq p C_{\text {Per }}\|u\|_{p}^{p-1},
\end{gathered}
$$

(5) and for all $u \in L^{p}(\Omega)$ and all $\alpha \geq 0$

$$
|\mathcal{F}(\alpha u)| \leq \alpha^{p} C_{\text {Per }}\|u\|_{p}^{p}
$$

Remark 2.3. (1) If $f(x, \cdot)$ is continuous for a.e. $x \in \Omega$, by (2.13) Clarke's generalized gradient $\partial \mathcal{F}(u)$ reduces to a singleton $\partial \mathcal{F}(u)=\left\{u^{*}\right\}$ with

$$
u^{*}(x)=f(x, u(x)) \quad \text { for a.e. } x \in \Omega \text {. }
$$

In this sense the generalized gradient $\partial \mathcal{F}(u)$ extends the classical Nemytskii operator $u \mapsto f(\cdot, u(\cdot))$ as formally used in (2.1) and (2.6) .

(2) Let us mention that the theorem remains true for $\Omega \subseteq \mathbb{R}^{n}$ merely open.

(3) With perturbations $\mathcal{E}_{\mathrm{Per}}$ and $\mathcal{G}_{\mathrm{Per}}$ of the form (2.9), our main results stated in Section 3 do not need further conditions than (f1)-(f3) for the integrand $f$ of $\mathcal{E}_{\mathrm{Per}}$, but our verification of the (epi)-condition requires a slightly stronger assumption on the integrand $g$ of $\mathcal{G}_{\mathrm{Per}}$ (cf. (3.16) below).

\section{Main results}

In this section we state the main results while the essential proofs are postponed to Section 5 and some preliminary results are presented in Section 4 . We always assume that $\Omega \subseteq \mathbb{R}^{n}$ is open and bounded with Lipschitz boundary.

\subsection{Perturbation of the energy}

For $\alpha>0$ and $1<p<\frac{n}{n-1}$ we investigate the perturbed eigenvalue problem of the 1-Laplace operator

$$
\begin{gathered}
\mathcal{E}_{T V}(v)+\mathcal{E}_{\mathrm{Per}}(v) \rightarrow \underset{v \in L^{p}(\Omega)}{\operatorname{Min} !} \\
\mathcal{G}_{1}(v)=\alpha .
\end{gathered}
$$

(cf. (2.2), (2.3)). 
Recall that we defined eigenfunctions to be critical points of

$$
\mathcal{E}_{T V}+\mathcal{E}_{\mathrm{Per}}+I_{\left\{\mathcal{G}_{1}=\alpha\right\}}
$$

on $L^{p}(\Omega)$. For the perturbation function $\mathcal{E}_{\mathrm{Per}}: L^{p}(\Omega) \rightarrow \mathbb{R}$ we assume that

(E1) $\mathcal{E}_{\text {Per }}$ is locally Lipschitz continuous on $L^{p}(\Omega)$,

$(\mathrm{E} 2) \mathcal{E}_{\text {Per }}$ is even, i.e. $\mathcal{E}_{\text {Per }}(v)=\mathcal{E}_{\text {Per }}(-v)$ for all $v \in L^{p}(\Omega)$,

(E3) there is a constant $C_{\text {Per }}>0$ such that for all $v \in L^{p}(\Omega)$

$$
\left|\mathcal{E}_{\mathrm{Per}}(v)\right| \leq C_{\mathrm{Per}}\|v\|_{p}^{p}
$$

(E4) for all $v^{*} \in \partial \mathcal{E}_{\mathrm{Per}}(v)$ and all $v \in L^{p}(\Omega)$ one has

$$
\left\|v^{*}\right\|_{p^{\prime}} \leq p C_{\mathrm{Per}}\|v\|_{p}^{p-1}
$$

Notice that all these conditions are fulfilled in the case where

$$
\mathcal{E}_{\text {Per }}(v)=\int_{\Omega} \int_{0}^{v(x)} f(x, s) \mathrm{d} s \mathrm{~d} x
$$

and the integrand $f$ satisfies (f1)-(f3) (cf. Theorem 2.2 above).

Since $\mathcal{E}_{T V}$ is lower semicontinuous on $L^{p}(\Omega)$, the functional $\mathcal{E}_{T V}+\mathcal{E}_{\text {Per }}$ turns out to be lower semicontinuous on $L^{p}(\Omega)$ too. By definition, $u \in L^{p}(\Omega)$ is an eigenfunction of our perturbed 1-Laplace problem if it is a critical point of (3.1), (3.2) in the sense of the weak slope, i.e. $|d \mathcal{E}|(u)=0$ for

$$
\mathcal{E}:=\mathcal{E}_{T V}+\mathcal{E}_{\mathrm{Per}}+I_{\left\{\mathcal{G}_{1}=\alpha\right\}}
$$

with $\alpha=\mathcal{G}_{1}(u)$. Let us first formulate some Euler-Lagrange equation as necessary condition for critical points of that problem. The proof can be found in Section 5.3 below.

Theorem 3.1 (Euler-Lagrange Equation). Let $\Omega \subseteq \mathbb{R}^{n}$ be open and bounded with Lipschitz boundary, let $1<p<\frac{n}{n-1}$, let $\mathcal{E}_{\mathrm{Per}}$ satisfy (E1)-(E4), and let $u$ be a critical point of variational problem (3.1), (3.2) for some $\alpha>0$. Then there exists a function $s \in L^{\infty}(\Omega)$ with

$$
s(x) \in \operatorname{Sgn}(u(x)) \quad \text { for a.e. } x \in \Omega,
$$

a vector field $z \in L^{\infty}\left(\Omega, \mathbb{R}^{n}\right)$ with

$$
\operatorname{div} z \in L^{p^{\prime}}(\Omega), \quad\|z\|_{\infty}=1, \quad \text { and } \quad \mathcal{E}_{T V}(u)=-\int_{\Omega} u \operatorname{div} z \mathrm{~d} x
$$


some $u^{*} \in \partial \mathcal{E}_{\mathrm{Per}}(u) \subseteq L^{p^{\prime}}(\Omega)$ and a Lagrange multiplier $\lambda \in \mathbb{R}$ such that the Euler-Lagrange equation

$$
-\operatorname{div} z+u^{*}=\lambda s \quad \text { on } \Omega
$$

is satisfied.

In the case where

$$
\mathcal{E}_{\mathrm{Per}}(v)=\int_{\Omega} \int_{0}^{v(x)} f(x, s) \mathrm{d} s \mathrm{~d} x
$$

with $f$ satisfying (f1)-(f3) we have

$$
u^{*}(x) \in\left[\underset{s \rightarrow u(x)}{\operatorname{ess} \inf _{s \rightarrow}} f(x, s), \underset{s \rightarrow u(x)}{\operatorname{ess} \sup } f(x, s)\right] \quad \text { for a.e. } x \in \Omega .
$$

If, in addition, $f(x, \cdot)$ is continuous for a.e. $x \in \Omega$, then $u^{*}(x)=f(x, u(x))$ a.e. on $\Omega$ and (3.5) becomes

$$
-\operatorname{div} z+f(x, u)=\lambda s \quad \text { on } \Omega
$$

Remark 3.2. (1) In contrast to the differentiable case of the p-Laplace operator with $p>1$, we cannot expect that the contrary of Theorem 3.1 is true, since a function u satisfying the Euler-Lagrange equation (3.5) doesn't need to be a a critical point of (3.1), (3.2). This fact is already known for the unperturbed case $f=0$ (cf. [1' $]$ ]).

(2) Using the eigenfunction $u$ as a test function in (3.5), we obtain for the corresponding eigenvalue

$$
\lambda=\frac{\mathcal{E}_{T V}(u)+\left\langle u^{*}, u\right\rangle}{\alpha}
$$

for some $u^{*} \in \partial \mathcal{E}_{\mathrm{Per}}(u)$. In the unperturbed case where $f=0$ and thus $u^{*}=0$, we have $\lambda=\frac{\mathcal{E}_{T V}(u)}{\alpha}$. Hence the eigenvalue $\lambda$ is uniquely determined by the eigenfunction $u$, though the functions $(z, s)$ in (3.5) related to u might be not unique (for the first eigenfunction of the 1-Laplace operator we even know that $(z, s)$ are not unique in general, cf. Kawohl $\&$ Schuricht [1]]). In the general perturbed situation it is not clear if the eigenvalue $\lambda$ associated to an eigenfunction $u$ is uniquely determined. It might happen that there are solutions $\left(s_{1}, z_{1}, u_{1}^{*}, \lambda_{1}\right)$ and $\left(s_{2}, z_{2}, u_{2}^{*}, \lambda_{2}\right)$ of (3.5) , both related to eigenfunction $u$ but with different eigenvalues $\lambda_{1} \neq \lambda_{2}$. However, if $\mathcal{E}_{\mathrm{Per}}$ has the form (3.6), this can only occur in the irregular case when for all $x$ from a set $E$ of positive measure the function $f(x, \cdot)$ is not continuous. 
We know that the (unperturbed) eigenvalue problem for the 1-Laplace operator has a sequence of eigensolutions $\left(\lambda_{k, 1}, u_{k, 1}\right)$ that can be constructed by methods of Ljusternik-Schnirelman theory (cf. [15]). Since the underlying minimax principle has some robustness against perturbations, we now want to show that the perturbed eigenvalue problem (3.1), (3.2) has a sequence of eigensolutions $\left(\lambda_{k, \alpha}, u_{k, \alpha}\right)$ for each $\alpha>0$ sufficiently small.

In critical point theory the Palais-Smale or (PS)-condition ensures some compactness. In our nonsmooth context the lower semicontinuous function $\mathcal{E}: M \rightarrow$ $\mathbb{R} \cup\{\infty\}$ on the metric space $M$ is said to satisfy the (PS)-condition at level $c \in \mathbb{R}$ if any Palais-Smale sequence $\left(u_{j}\right)_{j}$, i.e. $\mathcal{E}\left(u_{j}\right) \rightarrow c$ and $|d \mathcal{E}|\left(u_{j}\right) \rightarrow 0$, admits a convergent subsequence. If $\mathcal{E}$ satisfies the (PS)-condition at all levels $c \in \mathbb{R}$, we simply say that $\mathcal{E}$ satisfies the (PS)-condition.

Proposition 3.3 ((PS)-condition). Let $\Omega \subseteq \mathbb{R}^{n}$ be open and bounded with Lipschitz boundary, let $1<p<\frac{n}{n-1}$, let $\mathcal{E}_{\mathrm{Per}}$ satisfy (E1)-(E4), and let $\alpha>0$. Moreover we assume that one of the following conditions holds:

$\left(\mathrm{E} 5{ }^{\prime}\right) \mathcal{E}_{\mathrm{Per}}$ is globally bounded from below on $L^{p}(\Omega)$ or

(E5") with the embedding constant $C_{\mathrm{BV}}$ of $W_{0}^{1,1}(\Omega)$ in $L^{\frac{n}{n-1}}(\Omega)$ (cf. (1.13)) we have

$$
p \leq 1+\frac{1}{n}
$$

and

$$
\alpha^{n-(n-1) p}<C_{\mathrm{Per}}^{-1} C_{\mathrm{BV}}^{-(p-1) n}
$$

Then the (PS)-condition is satisfied for $\mathcal{E}=\mathcal{E}_{T V}+\mathcal{E}_{\mathrm{Per}}+I_{\left\{\mathcal{G}_{1}=\alpha\right\}}$ on $L^{p}(\Omega)$.

Remark 3.4. Condition (E5') is trivially satisfied provided $\mathcal{E}_{\mathrm{Per}}$ is of the form (3.6) and the integrand $f$ is bounded from below on $\Omega \times[0, \infty)$. Assumption $p \leq 1+\frac{1}{n}$, or equivalently $n-(n-1) p \geq \frac{1}{n}$, implies that (3.9) can always be achieved for $\alpha$ sufficiently close to zero.

We use the genus as topological index for the minimax construction of critical points. As genus gen ${ }_{X} S$ of a symmetric $S \subseteq X \backslash\{0\}$ in a Banach space $X$ we define the least integer $k \in \mathbb{N}$ such that there exists an odd continuous map $\phi: S \rightarrow \mathbb{R}^{k} \backslash\{0\}$ and we set gen $_{X} S=\infty$ provided such a map doesn't exist at all (cf. [21, Chap. 44.3]).

Theorem 3.5 (Existence of eigensolutions). Let $\Omega \subseteq \mathbb{R}^{n}$ be open and bounded with Lipschitz boundary, let $1<p<\frac{n}{n-1}$, let $\alpha>0$, and let $\mathcal{E}_{\mathrm{Per}}$ satisfy (E1)(E4) and either (E5') or (E5"). Then there exists a sequence of eigenfunctions $\left( \pm u_{k, \alpha}\right)_{k}$ of the perturbed eigenvalue problem (3.1), (3.2) with $\mathcal{G}_{1}\left( \pm u_{k, \alpha}\right)=\alpha$ 
and where the corresponding critical values $c_{k, \alpha}=\mathcal{E}_{T V}\left( \pm u_{k, \alpha}\right)+\mathcal{E}_{\mathrm{Per}}\left( \pm u_{k, \alpha}\right)$ are characterized by

$$
c_{k, \alpha}=\inf _{S \in \mathscr{S}_{k}^{\alpha}} \sup _{v \in S} \mathcal{E}_{T V}(v)+\mathcal{E}_{\mathrm{Per}}(v)
$$

with

$$
\mathscr{S}_{k}^{\alpha}:=\left\{S \subseteq L^{p}(\Omega) \text { compact, symmetric } ; \mathcal{G}_{1}=\alpha \text { on } S, \operatorname{gen}_{L^{p}} S \geq k\right\}
$$

The sequence of critical values $\left(c_{k, \alpha}\right)_{k}$ is unbounded. For each $k \in \mathbb{N}$ there is some $\alpha_{1}>0$ such that the family of rescaled eigenfunctions $v_{k, \alpha}:=\frac{u_{k, \alpha}}{\left\|u_{k, \alpha}\right\|_{1}}$ is bounded in $B V(\Omega)$ for $0<\alpha \leq \alpha_{1}$ and any choice of eigenfunctions $u_{k, \alpha}$ having critical value $c_{k, \alpha}$. Moreover, the Euler-Lagrange equation (3.5) from Theorem 3.1 holds for any critical point $u_{k, \alpha}$.

The proof of Theorem 3.5 is given in Section 5.3 and essentially relies on a general existence result for critical points of lower semicontinuous functional stated in Theorem 4.1 in Section 4.1. Let us finally formulate the claimed bifurcation result.

Theorem 3.6 (Bifurcation). Let $\Omega \subseteq \mathbb{R}^{n}$ be open and bounded with Lipschitz boundary, let $1<p \leq 1+\frac{1}{n}$, let $\alpha>0$ such that (3.9) holds, and let $\mathcal{E}_{\text {Per }}$ satisfy (E1)-(E4). Moreover, let $\left(\lambda_{k, \alpha}, u_{k, \alpha}\right)_{k}$ be the eigensolutions of (3.1), (3.2) from Theorem 3.5 with corresponding critical values $c_{k, \alpha}$ and let $\left(\lambda_{k, 1}\right)_{k}$ be the eigenvalues of the (unperturbed) 1-Laplace operator according to (1.11). Then we have

$$
\lim _{\alpha \rightarrow 0} \frac{c_{k, \alpha}}{\alpha}=\lim _{\alpha \rightarrow 0} \lambda_{k, \alpha}=\lambda_{k, 1}
$$

for all $k \in \mathbb{N}$ and, hence, the eigenvalues $\lambda_{k, 1}$ of the unperturbed problem are bifurcation values of the perturbed problem (3.1), (3.2).

\subsection{Perturbation of the constraint}

For $\beta>0$ and $p>1$ we now consider perturbed eigenvalue problems of the 1-Laplace operator of the form

$$
\mathcal{E}_{T V}(v) \rightarrow \underset{v \in L^{p}(\Omega)}{\operatorname{Min} !}
$$

subject to

$$
\mathcal{G}_{1}(v)+\mathcal{G}_{\mathrm{Per}}(v)=\beta
$$

(cf. (2.7), (2.8) ) where eigenfunctions had been defined to be critical points of

$$
\mathcal{E}_{T V}+I_{\left\{\mathcal{G}_{1}+\mathcal{G}_{\text {Per }}=\beta\right\}}
$$


on $L^{p}(\Omega)$. For the perturbation $\mathcal{G}_{\mathrm{Per}}: L^{p}(\Omega) \rightarrow \mathbb{R}$ we assume that

(G0) $1<p<1+\frac{1}{n}$,

(G1) $\mathcal{G}_{\text {Per }}$ is locally Lipschitz continuous on $L^{p}(\Omega)$,

$(\mathrm{G} 2) \mathcal{G}_{\text {Per }}$ is even, i.e. $\mathcal{G}_{\text {Per }}(v)=\mathcal{G}_{\text {Per }}(-v)$ for all $v \in L^{p}(\Omega)$,

(G3) there exists a constant $C_{\mathrm{Per}}>0$ such that for all $v \in L^{p}(\Omega)$

$$
0 \leq \mathcal{G}_{\mathrm{Per}}(v) \leq C_{\mathrm{Per}}\|v\|_{p}^{p}
$$

(G4) for all $v^{*} \in \partial \mathcal{G}_{\mathrm{Per}}(v)$ and all $v \in L^{p}(\Omega)$ one has

$$
\left\|v^{*}\right\|_{p^{\prime}} \leq p C_{\mathrm{Per}}\|v\|_{p}^{p-1}
$$

(G5) for all $v^{*} \in \partial \mathcal{G}_{\mathrm{Per}}(v)$ and all $v \in L^{p}(\Omega)$ one has

$$
\left.\begin{array}{ll}
v^{*}(x)>-1 & \text { for } v(x)>0 \\
v^{*}(x)<1 & \text { for } v(x)<0
\end{array}\right\} \quad \text { for a.e. } x \in \Omega
$$

Condition (G5) is in particular needed for the (PS)-condition. Clearly (3.16) is equivalent to

$$
v^{*}(x) \frac{v(x)}{|v(x)|}>-1 \quad \text { for a.e. } x \in \Omega \text { with } v(x) \neq 0
$$

and, thus, (3.16) implies

$$
v^{*}(x) v(x)>-|v(x)| \quad \text { for a.e. } x \in \Omega \text { with } v(x) \neq 0 .
$$

Similar to the previous case, conditions (G1)-(G4) are satisfied in the case of the Nemytskii potential

$$
\mathcal{G}_{\mathrm{Per}}(v)=\int_{\Omega} \int_{0}^{v(x)} g(x, s) \mathrm{d} s \mathrm{~d} x
$$

if the integrand $g$ satisfies (f1)-(f3) and, in addition,

$$
G(x, t):=\int_{0}^{t} g(x, s) \mathrm{d} s \geq 0 \quad \text { for all } t \in \mathbb{R} \text { and a.e. } x \in \Omega
$$

(cf. Theorem 2.2). For (G5) it is sufficient to require that

$$
g(x, s)>-1 \quad \text { a.e. on } \Omega \times[0, \infty),
$$

since $g(x, \cdot)$ is odd by (f3). It is not difficult to show that (3.18) and convexity of $G(x, \cdot)$ imply $g \geq 0$ on $\Omega \times[0, \infty)$ and, thus, condition (3.16) is valid. Let 
us start with some Euler-Lagrange equation as necessary condition for critical points. The proof can be found in Section 5.4 below.

Theorem 3.7 (Euler-Lagrange equation). Let $\Omega \subseteq \mathbb{R}^{n}$ be open and bounded with Lipschitz boundary, let $1<p<1+\frac{1}{n}$, let $\mathcal{G}_{\text {Per }}$ satisfy (G1)-(G5), and let $u$ be a critical point of variational problem (3.12), (3.13) for some $\beta>0$. Then there is some $s \in L^{\infty}(\Omega)$ with

$$
s(x) \in \operatorname{Sgn}(u(x)) \quad \text { for a.e. } x \in \Omega,
$$

a vector field $z \in L^{\infty}\left(\Omega, \mathbb{R}^{n}\right)$ with

$$
\operatorname{div} z \in L^{p^{\prime}}(\Omega), \quad\|z\|_{\infty}=1, \quad \text { and } \quad \mathcal{E}_{T V}(u)=-\int_{\Omega} \operatorname{div} z u \mathrm{~d} x,
$$

some $u^{*} \in \partial \mathcal{G}_{\mathrm{Per}}(u) \subseteq L^{p^{\prime}}(\Omega)$ and a Lagrange multiplier $\lambda \in \mathbb{R}$ such that the Euler-Lagrange equation

$$
-\operatorname{div} z=\lambda\left(s+u^{*}\right) \quad \text { on } \Omega
$$

is satisfied. In the case where

$$
\mathcal{G}_{\mathrm{Per}}(v)=\int_{\Omega} \int_{0}^{v(x)} g(x, s) \mathrm{d} s \mathrm{~d} x
$$

with $g$ satisfying (f1)-(f3), (3.18), and (3.19), we have

$$
u^{*}(x) \in\left[\underset{s \rightarrow u(x)}{\operatorname{ess} \inf _{s \rightarrow u(x)}} g(x, s), \underset{s \rightarrow \sup }{\operatorname{ess} \sup } g(x, s)\right] \quad \text { for a.e. } x \in \Omega .
$$

If, in addition, $g(x, \cdot)$ is continuous for a.e. $x \in \Omega$, then $u^{*}(x)=g(x, u(x))$ a.e. on $\Omega$ and (3.20) becomes

$$
-\operatorname{div} z=\lambda(s+g(x, u)) \quad \text { on } \Omega \text {. }
$$

Remark 3.8. With eigenfunction $u$ as test function in (3.20), we get for the corresponding eigenvalue

$$
\lambda=\frac{\mathcal{E}_{T V}(u)}{\mathcal{G}_{1}(u)+\left\langle u^{*}, u\right\rangle}
$$

for some $u^{*} \in \partial \mathcal{G}_{\mathrm{Per}}(u)$. In the unperturbed case we have $\lambda=\frac{\mathcal{E}_{T V}(u)}{\beta}$ and the eigenvalue is uniquely determined by the eigenfunction. However, for the perturbed problem it is not clear whether the eigenvalue $\lambda$ is uniquely determined by the eigenfunction $u$ ( $c f$. also Remark 3.2 about the perturbation of the energy).

Next we formulate our main result about the existence of eigensolutions of the 
perturbed problem (3.12), (3.13).

Theorem 3.9 (Existence of eigensolutions). Let $\Omega \subseteq \mathbb{R}^{n}$ be open and bounded with Lipschitz boundary, let $1<p<1+\frac{1}{n}$, let $\beta>0$, and let $\mathcal{G}_{\mathrm{Per}}$ satisfy conditions (G1)-(G5). Then there exists a sequence of eigenfunctions $\left( \pm u_{k, \beta}\right)_{k}$ of the perturbed eigenvalue problem (3.12), (3.13) where the corresponding critical values $c_{k, \beta}=\mathcal{E}_{T V}\left( \pm u_{k, \beta}\right)$ are characterized by

$$
c_{k, \beta}=\inf _{S \in \mathscr{S}_{k}^{\beta}} \sup _{u \in S} \mathcal{E}_{T V}(u),
$$

with

$$
\begin{aligned}
& \mathscr{S}_{k}^{\beta}:=\left\{S \subseteq L^{p}(\Omega)\right. \text { compact, } \text { symmetric } \\
&\left.\qquad \mathcal{G}_{1}+\mathcal{G}_{\mathrm{Per}}=\beta \text { on } S, \operatorname{gen}_{L^{p}} S \geq k\right\} .
\end{aligned}
$$

The sequence of critical values $\left(c_{k, \beta}\right)_{k}$ is unbounded. For each $k \in \mathbb{N}$ the family of rescaled eigenfunctions $v_{k, \beta}:=\frac{u_{k, \beta}}{\left\|u_{k, \beta}\right\|_{1}}$ is bounded in $B V(\Omega)$ for $\beta>0$ from bounded sets (in particular as $\beta \rightarrow 0$ ) and any choice of eigenfunctions $u_{k, \beta}$ having critical value $c_{k, \beta}$. Moreover, the Euler-Lagrange equation (3.20) from Theorem 3.7 holds for any critical point $u_{k, \beta}$.

The proof of Theorem 3.9 is given in Section 5.4. It is again based on the general critical point Theorem 4.1 stated in Section 4.1. Finally we formulate the intended bifurcation result.

Theorem 3.10 (Bifurcation). Let $\Omega \subseteq \mathbb{R}^{n}$ be open and bounded with Lipschitz boundary, let $1<p<1+\frac{1}{n}$, let $\beta>0$, and let $\mathcal{G}_{\text {Per }}$ satisfy (G1)-(G5). Moreover, let $\left(\lambda_{k, \beta}, u_{k, \beta}\right)_{k}$ be the eigensolutions of (3.12), (3.13) from Theorem 3.9 with corresponding critical values $c_{k, \beta}$ and let $\left(\lambda_{k, 1}\right)_{k}$ be the eigenvalues of the (unperturbed) 1-Laplace operator according to (1.11). Then we have

$$
\lim _{\beta \rightarrow 0} \frac{c_{k, \beta}}{\beta}=\lim _{\beta \rightarrow 0} \lambda_{k, \beta}=\lambda_{k, 1}
$$

for all $k \in \mathbb{N}$ and, hence, the eigenvalues $\lambda_{k, 1}$ of the unperturbed problem are bifurcation values of the perturbed problem (3.12), (3.13).

\section{Preparation of the proofs}

Before we carry out the proofs of our main results, we provide some tools from nonsmooth critical point theory and some essential norm estimates. 


\subsection{Tools from nonsmooth critical point theory}

Our existence results for eigensolutions rely on nonsmooth critical point theory for merely lower semicontinuous functionals based on the weak slope. With Theorem 4.1 below we provide a modified version of the general Ljusternik-Schnirelman type theorem stated in Degiovanni \& Schuricht [8, Thm. 2.5]. Though several similar results can be found in the literature, we did not find a direct reference for the presented version. Therefore we give a self-contained proof for the convenience of the reader and to keep track for some technical details. For completeness we first introduce the notion of weak slope.

Let $(M, d)$ be a metric space, let $\mathcal{F}: M \rightarrow \mathbb{R}$ be a continuous functional, and let $B_{\delta}(u) \subseteq M$ be the open ball of radius $\delta$ around $u$. The weak slope $|d \mathcal{F}|(u) \in[0, \infty]$ at the point $u \in M$ is the supremum over all $\sigma \in[0, \infty)$ such that there exists some $\delta>0$ and a continuous function $\eta: B_{\delta}(u) \times[0, \delta] \rightarrow M$ with

$$
d(\eta(v, t), v) \leq t \quad \text { and } \quad \mathcal{F}(\eta(v, t)) \leq \mathcal{F}(v)-\sigma t
$$

for all $(v, t) \in B_{\delta}(u) \times[0, \delta]$. This notion extends the value of $\left\|\mathcal{F}^{\prime}(u)\right\|$ for a smooth function $\mathcal{F}$ to merely continuous functions on a metric space (cf. [7]). We thus call $u \in M$ a critical point of $\mathcal{F}$ in the sense of the weak slope provided $|d \mathcal{F}|(u)=0$.

In a consistent way we extend the weak slope to a lower semicontinuous function $\mathcal{F}: M \rightarrow \mathbb{R}$ by means of the epigraph

$$
\operatorname{epi}(\mathcal{F}):=\{(v, t) \in M \times \mathbb{R} ; \mathcal{F}(v) \leq t\}
$$

equipped with the metric

$$
\tilde{d}\left(\left(v_{1}, t_{1}\right),\left(v_{2}, t_{2}\right)\right):=\sqrt{d\left(v_{1}, v_{2}\right)^{2}+\left(t_{1}-t_{2}\right)^{2}} .
$$

Using the projection $\mathscr{G}_{\mathcal{F}}:$ epi $(\mathcal{F}) \rightarrow \mathbb{R}$ given by $\mathscr{G}_{\mathcal{F}}(v, t):=t$, we define

$$
|d \mathcal{F}|(u):= \begin{cases}\frac{\left|d \mathscr{G}_{\mathcal{F}}\right|(u, \mathcal{F}(u))}{\sqrt{1-\left(\left|d \mathscr{G}_{\mathcal{F}}\right|(u, \mathcal{F}(u))\right)^{2}}} & \text { if }\left|d \mathscr{G}_{\mathcal{F}}\right|(u, \mathcal{F}(u))<1, \\ \infty & \text { if }\left|d \mathscr{G}_{\mathcal{F}}\right|(u, \mathcal{F}(u))=1\end{cases}
$$

This way the weak slope of $\mathcal{F}$ is traced back to the weak slope of the continuous function $\mathscr{G}_{\mathcal{F}}: \operatorname{epi}(\mathcal{F}) \rightarrow \mathbb{R}$.

In order to rule out possible critical points $(u, t)$ of $\mathscr{G}_{\mathcal{F}}$ with $t>\mathcal{F}(u)$, we assume the so-called epigraph (or short (epi)-) condition, i.e. for each $b>0$ we 
assume to have

$$
\inf \left\{\left|d \mathscr{G}_{\mathcal{F}}\right|(u, t) ;(u, t) \in \operatorname{epi}(\mathcal{F}), \mathcal{F}(u)<t,|t|<b\right\}>0 .
$$

Now we are able to state the general critical point theorem for even and lower semicontinuous functionals where we use the genus as topological index.

Theorem 4.1. Let $X$ be a real Banach space and let $\mathcal{F}: X \rightarrow \mathbb{R} \cup\{\infty\}$ be such that

(F1) $\mathcal{F}$ is lower semicontinuous, even (i.e. $\mathcal{F}(u)=\mathcal{F}(-u)$ ), and $F(0)=\infty$,

(F2) $\mathcal{F}$ is bounded from below,

(F3) $\mathcal{F}$ satisfies the (PS)-condition,

(F4) $\mathcal{F}$ satisfies the (epi)-condition, and

(F5) for all $k \in \mathbb{N}$ there exists $\Phi: \mathbb{S}^{k-1} \rightarrow X$ bijective, continuous and odd (i.e. $\Phi(-x)=-\Phi(x))$ with

$$
\sup \left\{\mathcal{F}(\Phi(x)) ; x \in \mathbb{S}^{k-1}\right\}<\infty
$$

where $\mathbb{S}^{k-1}$ denotes the $(k-1)$-dimensional sphere in $\mathbb{R}^{k}$.

Then there exists a sequence of pairs $\pm u_{1}, \pm u_{2}, \ldots$ of critical points of $\mathcal{F}$ with corresponding critical values $c_{k}=\mathcal{F}\left( \pm u_{k}\right), k \in \mathbb{N}$, given by

$$
c_{k}=\inf _{S \in \mathscr{S}_{k}} \sup _{v \in S} \mathcal{F}(v)
$$

where

$$
\mathscr{S}_{k}:=\{S \subseteq X \backslash\{0\} \text { symmetric and compact; gen } S \geq k\} \text {. }
$$

If the sublevel sets $\{\mathcal{F} \leq \gamma\}$ are compact for any $\gamma \in \mathbb{R}$, then

$$
c_{k} \rightarrow \infty \text { as } k \rightarrow \infty
$$

there is a set $S \in \mathscr{S}_{k}$ attaining the infimum in (4.3)

$$
c_{k}=\sup _{v \in S} \mathcal{F}(v)
$$

and any $S \in \mathscr{S}_{k}$ with (4.4) contains a critical point $\tilde{u}_{k}$ with critical value $c_{k}$.

The proof of the theorem will be given in Section 5.2 below.

Remark 4.2. (1) With minor modifications we could use Theorem 2.5 from [8] to get the existence of a sequence of critical points as in the previous 
theorem. However, the unboundedness of the $c_{k}$ and their minimax characterization as in (4.3) is not stated there. The unboundedness of the $c_{k}$ could probably also be derived from Degiovanni \& Marzocchi [1], Thm. 3.10], but there the category is used as topological index. Though it is well known that the genus of a closed symmetric set equals its category in the projective space where antipodal points are identified (cf. Rabinowitz [20, Thm. 3.7] and Fadell [11, p. 40]), critical point theory for merely lower semicontinuous functionals $\mathcal{F}$ is reduced to the investigation of the continuous functional $\mathscr{G}_{\mathcal{F}}$ where some rather technical arguments are needed to verify that the critical values obtained with the concept of category agree with that obtained by using the genus (cf. Littig \& Schuricht [14, Cor. 2.2] and its proof).

(2) The situation of the theorem might be covered by the abstract results of Corvellec [4], but it is not immediate and might be quite technical to deduce the desired statements.

(3) If condition (F5) is satisfied not for all $k \in \mathbb{N}$ but only for some $k_{0} \in \mathbb{N}$ (e.g. if $X$ is finite dimensional), it is not difficult to adapt our proof to show that there exist at least $k_{0}$ pairs of critical points $\pm u_{1}, \ldots, \pm u_{k_{0}}$ with corresponding critical values given by (4.3).

(4) Notice that, in general, there might be critical points of $\mathcal{F}$ with critical level $c_{k}$ that do not belong to some $S \in \mathscr{S}_{k}$ satisfying (4.4).

\subsection{Norm estimates}

Here we derive some norm estimates needed for our convergence results.

Proposition 4.3. Let $\Omega \subseteq \mathbb{R}^{n}$ be open and bounded, let $p \in\left[1, \frac{n}{n-1}\right]$, and let $C_{\mathrm{BV}}$ be the embedding constant of $W_{0}^{1,1}(\Omega)$ in $L^{\frac{n}{n-1}}(\Omega)$ (cf. (1.13) ). Then we have

$$
\|u\|_{p} \leq C_{\mathrm{BV}}^{\frac{n(p-1)}{p}}\|u\|_{1}^{1-\frac{n(p-1)}{p}}\|D u\|_{1}^{\frac{n(p-1)}{p}} \text { for all } u \in W_{0}^{1,1}(\Omega) .
$$

Proof. For $u \in L^{\frac{n}{n-1}}(\Omega)$ the interpolation inequality tells us that

$$
\|u\|_{p} \leq\|u\|_{1}^{\theta}\|u\|_{\frac{n}{n-1}}^{1-\theta}
$$

with

$$
\frac{1}{p}=\frac{\theta}{1}+\frac{1-\theta}{n /(n-1)} \quad \text { or, equivalently, } \quad \theta=1-\frac{n(p-1)}{p} .
$$

Then the assertion directly follows with (1.13). 
Consequently we can control $\|u\|_{p}$ by joint knowledge of $\|u\|_{1}$ and $\|D u\|_{1}$. Since $\|D u\|_{1}=\mathcal{E}_{T V}(u)$ for $u \in W_{0}^{1,1}(\Omega)$, the following statement for $B V$ functions is not surprising.

Corollary 4.4. Let $\Omega \subseteq \mathbb{R}^{n}$ be open and bounded with Lipschitz boundary, let $p \in$ $\left[1, \frac{n}{n-1}\right]$, let $C_{\mathrm{BV}}$ be the embedding constant of $W_{0}^{1,1}(\Omega)$ in $L^{\frac{n}{n-1}}(\Omega)$ (cf. (1.13) ), and let $\mathcal{E}_{T V}$ be as in (1.14). Then

$$
\|u\|_{p}^{p} \leq C_{\mathrm{BV}}^{(p-1) n}\|u\|_{1}^{n-(n-1) p} \mathcal{E}_{T V}(u)^{(p-1) n} \quad \text { for all } u \in B V(\Omega) .
$$

If additionally $p \leq \frac{n+1}{n}$, we have

$$
\|u\|_{p}^{p} \leq C_{\mathrm{BV}}^{(p-1) n}\|u\|_{1}^{n-(n-1) p}\left(\mathcal{E}_{T V}(u)+1\right) \quad \text { for all } u \in B V(\Omega) .
$$

Proof. The fist estimate follows by taking the $p$-th power of the inequality in Proposition 4.3 and by approximating $\mathcal{E}_{T V}(u)$ as in Theorem 3.1 of $[14]$. For the second estimate we observe that $t^{(p-1) n} \leq 1+t$ for $t \geq 0$ by $(p-1) n \leq 1$ and then we set $t=\mathcal{E}_{T V}(u)$.

Notice that (4.5) allows to control the $p$-th order growth of $\|u\|_{p}^{p}$ by the first order growth of $\mathcal{E}_{T V}(u)$ provided $\|u\|_{1}$ is known to be bounded.

\section{Proofs of the main results}

We first present the proof of Theorem 2.2 about properties of integral functionals we have in mind as perturbations. Then the general Theorem 4.1 about existence of critical points is verified. In Section 5.3 proofs related to perturbations of the energy are given and, finally, Section 5.4 collects the proofs related to perturbations of the constraint.

\subsection{Proof of Theorem 2.2}

Proof of Theorem 2.2. If $\mathcal{F}$ is well-defined, then antisymmetry of $f(x, \cdot)$ as in (2.12) implies that $\mathcal{F}$ is symmetric, i.e. $\mathcal{F}(u)=\mathcal{F}(-u)$, and we have

$$
\mathcal{F}(u)=\int_{\Omega} \int_{0}^{u(x)} f(x, s) \mathrm{d} s \mathrm{~d} x=\int_{\Omega} \int_{0}^{|u(x)|} f(x, s) \mathrm{d} s \mathrm{~d} x=\mathcal{F}(|u|) .
$$

Let us now verify that $\mathcal{F}$ is well-defined. By (f1) function $F: \Omega \times[0, \infty) \rightarrow \mathbb{R}$ with

$$
F(x, t):=\int_{0}^{t} f(x, s) \mathrm{d} s
$$


is a Carathéodory function (which includes that $F$ is well defined) and, hence, $F(\cdot, u(\cdot))$ is measurable on $\Omega$ for any measurable $u$.

We now take $u, w \in L^{p}(\Omega)$ with $\|u\|_{p},\|w\|_{p} \leq R$. Then $(|u|+|w|)^{p-1} \in L^{p^{\prime}}(\Omega)$ with

$$
\left\|(|u|+|w|)^{p-1}\right\|_{p^{\prime}}^{p^{\prime}}=\|(|u|+|w|)\|_{p}^{p} \leq(2 R)^{p} .
$$

By (5.1), (2.11), and Hölder's inequality we get

$$
\begin{aligned}
|\mathcal{F}(u)-\mathcal{F}(w)| & =\left|\int_{\Omega} \int_{|w(x)|}^{|u(x)|} f(x, s) \mathrm{d} s \mathrm{~d} x\right| \\
& \leq p C_{\mathrm{Per}} \int_{\Omega}|| u(x)|-| w(x)||(|u(x)|+|w(x)|)^{p-1} \mathrm{~d} x \\
& \leq p C_{\mathrm{Per}}\|u-w\|_{p}\left\|(|u|+|w|)^{p-1}\right\|_{p^{\prime}} \\
& \leq p C_{\mathrm{Per}}(2 R)^{p-1}\|u-w\|_{p} .
\end{aligned}
$$

Since $\mathcal{F}(w)=0$ for $w=0$, we readily obtain that $\mathcal{F}(u)$ is finite for all $u \in L^{p}(\Omega)$. Moreover $\mathcal{F}$ is uniformly Lipschitz continuous on bounded subsets of $L^{p}(\Omega)$.

A straightforward calculation using (2.11) gives for $\alpha \geq 0$ that

$$
\begin{aligned}
|\mathcal{F}(\alpha u)| & \leq \int_{\Omega}\left|\int_{0}^{\alpha u(x)} f(x, s) \mathrm{d} s\right| \mathrm{d} x \\
& \leq \int_{\Omega}\left|\int_{0}^{|\alpha u(x)|} p C_{\mathrm{Per}} s^{p-1} \mathrm{~d} s\right| \mathrm{d} x \\
& =\int_{\Omega} C_{\mathrm{Per}}|\alpha u|^{p} \mathrm{~d} x \\
& =C_{\mathrm{Per}} \alpha^{p}\|u\|_{p}^{p}
\end{aligned}
$$

i.e. we have shown (2.15).

It remains to prove assertion (4) about $\partial \mathcal{F}(u)$. For $u, v \in L^{p}(\Omega), u^{*} \in \partial \mathcal{F}(u)$, and with the notation

$$
F_{x}(t):=F(x, t),
$$

we derive

$$
\begin{aligned}
\int_{\Omega} u^{*} v \mathrm{~d} x \leq \mathcal{F}^{0}(u ; v) & =\limsup _{w \rightarrow u, t \downarrow 0} \frac{\mathcal{F}(w+t v)-\mathcal{F}(w)}{t} \\
& =\limsup _{w \rightarrow u, t \downarrow 0} \int_{\Omega} \frac{F_{x}(w(x)+t v(x))-F_{x}(w(x))}{t} \mathrm{~d} x .
\end{aligned}
$$

Notice that $F_{x}$ is the primitive of a locally bounded function for a.e. $x \in \Omega$ 
by (2.11). Hence we are in the situation of Example 2.2.5 from [3] and obtain that $F_{x}$ is locally Lipschitz continuous with

$$
\partial F_{x}(t)=[\underset{s \rightarrow t}{\operatorname{essinf}} f(x, s), \underset{s \rightarrow t}{\operatorname{ess} \sup } f(x, s)] \quad \text { for } t \in \mathbb{R} .
$$

Again by (2.11) we get

$$
\left|F^{*}\right| \leq p C_{\mathrm{Per}}|t|^{p-1} \quad \text { for all } F^{*} \in \partial F_{x}(t) .
$$

Let us now choose a sequence $\left(w_{k}\right)_{k}$ with $w_{k} \rightarrow u$ in $L^{p}(\Omega)$ and $t_{k} \downarrow 0$ with $t_{k} \leq 1$ such that

$$
\mathcal{F}^{0}(u ; v)=\lim _{k \rightarrow \infty} \int \frac{F_{x}\left(w_{k}(x)+t_{k} v(x)\right)-F_{x}\left(w_{k}(x)\right)}{t_{k}} \mathrm{~d} x .
$$

Without loss of generality we may assume that $w_{k}(x) \rightarrow u(x)$ a.e. on $\Omega$. By Lebourg's Theorem (cf. [3], Thm. 2.3.7]) we have that for a.e. $x \in \Omega$ and every $k \in \mathbb{N}$ there is some $\theta \in(0,1)$ and $F_{k}^{*}(x) \in \partial F_{x}\left(w_{k}(x)+\theta t_{k} v(x)\right)$ such that

$$
\begin{aligned}
\left|F_{x}\left(w_{k}(x)+t_{k} v(x)\right)-F_{x}\left(w_{k}(x)\right)\right| & =\left|F_{k}^{*}(x)\left(w_{k}(x)+t_{k} v(x)-w_{k}(x)\right)\right| \\
& =t_{k}\left|F_{k}^{*}(x) v(x)\right| \\
& \leq p C_{\mathrm{Per}}\left(\left|w_{k}(x)\right|+|v(x)|\right)^{p-1}|v(x)|
\end{aligned}
$$

by (5.5). Obviously

$$
h_{k}:=\left|w_{k}\right|+|v| \rightarrow|u|+|v| \quad \text { in } L^{p}(\Omega)
$$

and the nonlinear operator $J_{p}: L^{p}(\Omega) \rightarrow L^{p^{\prime}}(\Omega)$ given by

$$
J_{p}(u)(x):=|u(x)|^{p-1} \operatorname{sgn}(u(x))
$$

is a homeomorphism (cf. [2, p. 72]). Thus

$$
h_{k}^{*}:=J_{p}\left(h_{k}\right) \rightarrow J_{p}(|u|+|v|) \quad \text { in } L^{p^{\prime}}(\Omega) .
$$

Whence

$$
g_{k}:=h_{k}^{*}|v| \rightarrow g:=(|u|+|v|)^{p-1}|v| \quad \text { in } L^{1}(\Omega)
$$

and, by assumption, also pointwise a.e. on $\Omega$. Picking an appropriate subsequence if necessary we may assume that $\sum_{k \in \mathbb{N}}\left\|g_{k}-g\right\|_{1}<\infty$. Then $g+\sum_{k \in \mathbb{N}}\left|g_{k}\right|$ is a majorant of all $g_{k}$ and also of all integrands in (5.7). Therefore, by Fatou's 
Lemma, (5.6) implies

$$
\int_{\Omega} u^{*}(x) v(x) \mathrm{d} x \leq \mathcal{F}^{0}(u ; v) \leq \int_{\Omega} \limsup _{k \rightarrow \infty} \frac{F_{x}\left(w_{k}(x)+t_{k} v(x)\right)-F_{x}\left(w_{k}(x)\right)}{t_{k}} \mathrm{~d} x .
$$

Note that the integrand on the right hand side is bounded by $F_{x}^{0}(u(x) ; v(x))$ for a.e. $x \in \Omega$. Since the argument holds true for all $v \in L^{p}(\Omega)$, we can choose $v=t \chi_{E}$ for appropriate $E \subseteq \Omega$ and $t \in \mathbb{R}$ to obtain

$$
u^{*}(x) t \leq F_{x}^{0}(u(x) ; t) \quad \text { for all } t \in \mathbb{R} \text { and a.e. } x \in \Omega \text {. }
$$

Consequently, by definition,

$$
u^{*}(x) \in \partial F_{x}(u(x)) \text { for a.e. } x \in \Omega
$$

and with (5.4) we have verified (2.13). By (2.11) we thus obtain

$$
\begin{aligned}
& \left\|u^{*}\right\|_{p^{\prime}}^{p^{\prime}} \leq \int_{\Omega} \max \{|\underset{s \rightarrow u(x)}{\operatorname{essinf}} f(x, s)|,|\underset{s \rightarrow u(x)}{\operatorname{ess} \sup } f(x, s)|\}^{p^{\prime}} \mathrm{d} x \\
& \leq \int_{\Omega}\left(p C_{\mathrm{Per}}\right)^{p^{\prime}}|u(x)|^{p} \mathrm{~d} x \\
& =\left(p C_{\mathrm{Per}}\right)^{p^{\prime}}\|u\|_{p}^{p}
\end{aligned}
$$

and (2.14) follows.

\subsection{Proof of Theorem 4.1}

Proof of Theorem 4.1. It is well known that (F5) ensures the classes $\mathscr{S}_{k}$ to be nonempty (cf. [21, Chap. 44.3]) and $c_{k}<\infty$. Thus, by boundedness of $\mathcal{F}$ from below, the values $c_{k}$ in (4.3) are finite.

If a set $\tilde{S} \subseteq \operatorname{epi}(\mathcal{F})$ has the property that $(u, s) \in \tilde{S}$ implies $(-u, s) \in \tilde{S}$, we define the genus gen $\operatorname{sen}_{1}$ of $\tilde{S}$ as the genus of the projection of $\tilde{S}$ on the first coordinate, i. e.

$$
\operatorname{gen}_{1} \tilde{S}:=\operatorname{gen}\{u \in X ;(u, s) \in \tilde{S}\}
$$

Taking

$$
\begin{gathered}
\tilde{\mathscr{S}}_{k}:=\left\{\tilde{S} \subseteq \operatorname{epi}(\mathcal{F}) ; \tilde{S} \text { compact, } \operatorname{gen}_{1} \tilde{S} \geq k,(-u, s) \in \tilde{S} \forall(u, s) \in \tilde{S}\right\}, \\
\tilde{c}_{k}:=\inf _{\tilde{S} \in \tilde{\mathscr{S}}_{k}} \sup _{(u, s) \in \tilde{S}} \mathscr{G}_{\mathcal{F}}(u, s)
\end{gathered}
$$

we have

$$
c_{k}=\tilde{c}_{k} .
$$


Indeed, invoking the definition of $\mathscr{G}_{\mathcal{F}}$ and $\tilde{S} \subseteq \operatorname{epi}(\mathcal{F})$, we see that the value $\tilde{c}_{k}$ does not change if we restrict our attention to sets $\tilde{S} \in \tilde{\mathscr{S}}_{k}$ of the form

$$
\tilde{S}=S \times\left\{\sup _{u \in S} \mathcal{F}(u)\right\}
$$

with $S \in \mathscr{S}_{k}$. We may assume that $\sup _{u \in S} \mathcal{F}(u)<\infty$ by (F5) and, hence, for those sets $\tilde{S}$ the equality is immediate.

We define the set of critical points of $\mathcal{F}$ at level $c$ by

$$
K_{c}:=\{u \in X ; \mathcal{F}(u)=c \text { and }|d \mathcal{F}|(u)=0\}
$$

Let us assume that $c_{k}$ is not a critical value, i.e. $K_{c_{k}}=\emptyset$. We will show that then there is some $\tilde{\varepsilon}>0$ such that

$$
K_{c}=\emptyset \quad \text { for all } \quad c \in\left(c_{k}-\tilde{\varepsilon}, c_{k}+\tilde{\varepsilon}\right) .
$$

If this is not true, we find a sequence of critical points $\left(u_{j}\right)_{j}$ of the function $\mathcal{F}$ with $\mathcal{F}\left(u_{j}\right) \rightarrow c_{k}$. Then, by definition, $\left(u_{j}, \mathcal{F}\left(u_{j}\right)\right)_{j}$ is a sequence of critical points of the continuous function $\mathscr{G}_{\mathcal{F}}: \operatorname{epi}(\mathcal{F}) \rightarrow \mathbb{R}$. Since $\left(u_{j}\right)_{j}$ is a PalaisSmale sequence for $\mathcal{F}$, it admits a convergent subsequence (denoted the same way) with $u_{j} \rightarrow: u$. By lower semicontinuity of $\mathcal{F}$ we have $\mathcal{F}(u) \leq c_{k}$. Since the weak slope is lower semicontinous with respect to the graph metric (see [7, Prop. 2.6]), we obtain that $\left(u, c_{k}\right)=\lim _{j \rightarrow \infty}\left(u_{j}, \mathcal{F}\left(u_{j}\right)\right)$ is a critical point of $\mathscr{G}_{\mathcal{F}}$. From the (epi)-condition (4.2) we derive that $c_{k}=\mathcal{F}(u)$ and, therefore, $u \in K_{c_{k}}$. But this is a contradiction and verifies (5.8).

According to the first part of the proof of Theorem 2.5 in [8] (applied with $\left.f=\mathscr{G}_{\mathcal{F}}, X=\operatorname{epi}(\mathcal{F}), \Phi(u, s)=(-u, s), \mathcal{O}=\emptyset\right)$ there is some $\varepsilon \in(0, \tilde{\varepsilon}]$ and a continuous map $\eta: \operatorname{epi}(\mathcal{F}) \times[0,1] \rightarrow \operatorname{epi}(\mathcal{F})$ such that for all $(u, s) \in \operatorname{epi}(\mathcal{F})$, all $t \in[0,1]$, and with the epigraph metric $\tilde{d}$ as in (4.1)

$$
\begin{aligned}
\tilde{d}(\eta((u, s), t),(u, s)) & \leq t \\
s \notin\left[c_{k}-\tilde{\varepsilon}, c_{k}+\tilde{\varepsilon}\right] & \Rightarrow \eta((u, s), t)=(u, s) \\
\eta\left(\left\{\mathscr{G}_{\mathcal{F}} \leq c_{k}+\varepsilon\right\}, 1\right) & \subseteq\left\{\mathscr{G}_{\mathcal{F}} \leq c_{k}-\varepsilon\right\} \\
\eta((-u, s), t) & =-\eta((u, s), t) .
\end{aligned}
$$

By (4.3) there is $S_{1} \in \mathscr{S}_{k}$ such that

$$
\sup _{u \in S_{1}} \mathcal{F}(u) \leq c_{k}+\varepsilon
$$


For $a:=\sup _{u \in S_{1}} \mathcal{F}(u)$ we define $\eta_{1}: X \rightarrow X \times\{a\}$ by

$$
\eta_{1}(u):=(u, a) \quad \text { and } \quad T_{1}:=\eta_{1}\left(S_{1}\right)=S_{1} \times\{a\} \subseteq \operatorname{epi}(\mathcal{F}) .
$$

With $\eta$ from above we consider

$$
T_{2}:=\eta\left(T_{1}, 1\right) \subseteq \operatorname{epi}(\mathcal{F})
$$

By (5.9) we have $s \leq c_{k}-\varepsilon$ for all $(u, s) \in T_{2}$. Let $\eta_{2}: \operatorname{epi}(\mathcal{F}) \rightarrow X$ denote the projection given by

$$
\eta_{2}(u, s):=u \quad \text { and with } \quad S_{2}:=\eta_{2}\left(T_{2}\right)
$$

we then obtain

$$
\sup _{u \in S_{2}} \mathcal{F}(u) \leq \sup _{(u, s) \in T_{2}} s \leq c_{k}-\varepsilon
$$

The set $S_{2}$ is obtained as continuous image of $S_{1}$ under $\eta_{2} \circ \eta \circ \eta_{1}$ and thus compact. By (5.10) we see that $\eta_{2} \circ \eta \circ \eta_{1}$ is odd and, thus, an elementary property of genus gives

$$
\text { gen } S_{2} \geq \text { gen } S_{1} \text {. }
$$

Consequently, $S_{2} \in \mathscr{S}_{k}$ and (5.12) contradicts the definition (5.9) of $c_{k}$. Hence our assumption $K_{c_{k}}=\emptyset$ must be wrong and $c_{k}$ has to be a critical level for any $k \in \mathbb{N}$.

For the proof of the remaining assertions let $\{\mathcal{F} \leq \gamma\}$ be compact for any $\gamma \in \mathbb{R}$. Here we also use a compactness result of Blaschke (cf. [1, Thm. 4.4.15]) saying that the set $\mathcal{K}$ of nonempty compact subsets of a compact metric space $(K, d)$ is compact provided $\mathcal{K}$ is equipped with the Hausdorff distance

$$
d_{H}\left(K_{1}, K_{2}\right):=\sup _{x \in K_{1}} d\left(x, K_{2}\right)+\sup _{x \in K_{2}} d\left(x, K_{1}\right)
$$

Moreover, if $K_{j} \rightarrow K_{0}$ in the Hausdorff distance, then $x_{0} \in K_{0}$ if and only if for each $j \in \mathbb{N}$ there is some $x_{j} \in K_{j}$ such that $x_{j} \rightarrow x_{0}$ (cf. [1, Prop. 4.4.14]).

First we fix $k \in \mathbb{N}$ and choose a sequence $\left(S_{j}\right)$ in $\mathscr{S}_{k}$ with

$$
c_{k}=\lim _{j \rightarrow \infty} \sup _{v \in S_{j}} \mathcal{F}(v)
$$

We can assume that all $S_{j}$ belong to the compact set $\left\{\mathcal{F} \leq c_{k}+1\right\}$ and, by Blaschke's theorem, that they converge to some compact $S \subseteq\left\{\mathcal{F} \leq c_{k}+1\right\}$ with respect to the Hausdorff metric. The pointwise characterization of the limit and the lower semicontinuity of $\mathcal{F}$ imply that $S$ is symmetric, that $0 \notin S$ (recall 
$\mathcal{F}(0)=\infty)$, and that

$$
\sup _{v \in S} \mathcal{F}(v) \leq \lim _{j \rightarrow \infty} \sup _{v \in S_{j}} \mathcal{F}(v)=c_{k}
$$

By a standard property of genus there is an open neighborhood $U$ of $S$ with gen $S=$ gen $\bar{U}$ (cf. [21, Chap. 44.3]). The convergence $S_{j} \rightarrow S$ in the Hausdorff metric implies $S_{j} \subseteq U$ for $k$ large enough. Hence, the monotonicity of genus with respect to inclusions gives

$$
\text { gen } S \geq \limsup _{j \rightarrow \infty} \text { gen } S_{j} \geq k .
$$

Therefore $S \in \mathscr{S}_{k}$ and the definition of $c_{k}$ implies (4.4).

For fixed $k$ we now choose any $S \in \mathscr{S}_{k}$ satisfying $c_{k}=\sup _{v \in S} \mathcal{F}(v)$ and let us assume that

$$
S \cap K_{c_{k}}=\emptyset
$$

We show that there exists a neighborhood $\mathcal{U}$ of $S \times\left\{c_{k}\right\}$ in epi $(\mathcal{F})$ containing no critical points of $\mathscr{G}_{\mathcal{F}}$. Otherwise we find critical points $\left(v_{j}, t_{j}\right)$ of $\mathscr{G}_{\mathcal{F}}$ with $\left(v_{j}, t_{j}\right) \rightarrow\left(v, c_{k}\right)$ for some $v \in S$ (recall compactness of $S$ and thus $S \times\left\{c_{k}\right\}$ ). Since the weak slope is lower semicontinuous, $\left(v, c_{k}\right)$ is a critical point of $\mathscr{G}_{\mathcal{F}}$ and, by the (epi)-condition (F4), $v \in S$ is critical point of $\mathcal{F}$ with critical value $c_{k}$. But this contradicts (5.13) and verifies our claim. Consequently, by the compactness of $S \times\left\{c_{k}\right\}$, there is some open neighborhood $\mathcal{O}$ of the critical points of $\mathscr{G}_{\mathcal{F}}$ in $\operatorname{epi}(\mathcal{F})$ with

$$
\mathcal{O} \cap\left(S \times\left\{c_{k}\right\}\right)=\emptyset .
$$

According to Deformation Theorem 2.14 in [5], applied at critical value $c_{k}$, there exists a continuous map $\varphi: \operatorname{epi}(\mathcal{F}) \times[0,1] \rightarrow \operatorname{epi}(\mathcal{F})$ and some $\varepsilon>0$ such that

$$
\mathscr{G}_{\mathcal{F}}(\varphi((u, s), 1)) \leq c_{k}-\varepsilon \quad \text { for }(u, s) \in\left\{\operatorname{epi}(\mathcal{F}) \backslash \mathcal{O} ; \mathscr{G}_{\mathcal{F}}(u, s) \leq c_{k}+\varepsilon\right\}
$$

An easy adaption of the proof of [5, Thm. 2.17] shows that we can assume

$$
\varphi((u, s), t)=-\varphi((-u, s), t) \quad \text { for }(u, s) \in \operatorname{epi}(\mathcal{F}), t \in[0,1]
$$

With $\eta_{2}$ from (5.11) we get that

$$
\tilde{S}:=\eta_{2}\left(\varphi\left(S \times\left\{c_{k}\right\}, 1\right)\right)
$$


is symmetric and, as continuous image of a compact set, compact. Moreover

$$
\mathcal{F}(u) \leq c_{k}-\varepsilon \quad \text { for } u \in \tilde{S}
$$

We have gen $\tilde{S} \geq$ gen $S \geq k$, since a continuous map does not decrease the genus. Thus $\tilde{S} \in \mathscr{S}_{k}$ and (5.14) contradicts the definition of $c_{k}$. Consequently (5.13) must be wrong and $S$ contains a critical point $\tilde{u}_{k}$ with critical value $c_{k}$.

Finally let us assume that

$$
c:=\limsup _{k \rightarrow \infty} c_{k}<\infty .
$$

According to (4.4) we can choose $S_{k} \in \mathscr{S}_{k}$ with $c_{k}=\sup _{v \in S_{k}} \mathcal{F}(v)$. Since the $c_{k}$ are increasing, we can assume that all $S_{k}$ belong to the compact set $\{\mathcal{F} \leq c\}$ and, by Blaschke's Theorem, that the $S_{k}$ converge to some compact and symmetric set $\tilde{S} \subseteq\{\mathcal{F} \leq c\}$ in the Hausdorff metric. In particular $0 \notin \tilde{S}$ by $(\mathrm{F} 1)$. As above there is an open neighborhood $V$ of $\tilde{S}$ with gen $\bar{V}=$ gen $\tilde{S}$. Since $S_{k} \subseteq V$ for $k$ large enough, the monotonicity of genus with respect to inclusions implies

$$
k \leq \operatorname{gen} S_{k} \leq \operatorname{gen} \bar{V}=\operatorname{gen} \tilde{S} \text { for all } k \in \mathbb{N} .
$$

But this contradicts the fact that the genus of a compact set is finite. Therefore $\left(c_{k}\right)_{k}$ cannot be bounded and the proof is complete.

\subsection{Proofs for perturbations of the energy}

Proof of Theorem 3.1. We will apply [8, Cor. 3.7] with

$$
Y=L^{p}(\Omega), f_{0}=\mathcal{E}_{T V}, f_{1}=\mathcal{E}_{\mathrm{Per}}, g_{0}=-1, g_{1}=\mathcal{G}_{1}-\alpha
$$

Let $u \in B V(\Omega)$ with $\mathcal{G}_{1}(u)=\alpha$. In order to prove the (epi)-condition (cf. [8, Thm. 3.4]) we need to show that there are $\left.u_{1}, u_{2} \in B V(\Omega)\right)$ such that

$$
\mathcal{G}_{1}^{0}\left(u ; u_{1}-u\right)<0 \quad \text { and } \quad \mathcal{G}_{1}^{0}\left(u ; u-u_{2}\right)<0
$$

Recalling the generalized gradient of $\mathcal{G}_{1}$ (cf. [13, Prop. 4.23]) and using [3, Prop. 2.1.2] with $u_{1}=0$ and $u_{2}=2 u$, we derive

$$
\begin{aligned}
\mathcal{G}_{1}^{0}\left(u ; u_{1}-u\right) & =\mathcal{G}_{1}^{0}\left(u ; u-u_{2}\right)=\mathcal{G}_{1}^{0}(u ;-u) \\
& =\max _{u^{*} \in \partial \mathcal{G}_{1}(u)}\left\langle u^{*},-u\right\rangle=-\alpha<0 .
\end{aligned}
$$

The Euler-Lagrange equation (3.5) is now a consequence of [13, Prop. 4.23] and, for (3.7), we use the properties of $\partial \mathcal{E}_{\text {Per }}$ stated in Theorem 2.2 . 
Proof of Proposition 3.3. Let $c \in \mathbb{R}$ and let $\left(u_{j}\right)_{j}$ be a Palais-Smale sequence for the function $\mathcal{E}$, i.e. $\mathcal{E}\left(u_{j}\right) \rightarrow c$ and $|d \mathcal{E}|\left(u_{j}\right) \rightarrow 0$. In the case (E5') where $\mathcal{E}_{\text {Per }}$ is bounded from below by some $L \leq 0$, we eventually have

$$
\mathcal{E}_{T V}\left(u_{j}\right) \leq c+1-\mathcal{E}_{\mathrm{Per}}\left(u_{j}\right) \leq c+1-L
$$

Since $\mathcal{E}_{T V}$ is a norm on $B V(\Omega)$ equivalent to the standard norm, $\left(u_{j}\right)_{j}$ is bounded in $B V(\Omega)$. Thus, the compact embedding $B V(\Omega) \hookrightarrow L^{p}(\Omega)$ ensures the existence of a convergent subsequence in $L^{p}$ and the (PS)-condition is verified.

If condition (E5") is satisfied, we use (4.5) and $\left\|u_{j}\right\|_{1}=\alpha$ to estimate

$$
\begin{aligned}
c+1 & \geq \mathcal{E}_{T V}\left(u_{j}\right)+\mathcal{E}_{\mathrm{Per}}\left(u_{j}\right) \\
& \geq \mathcal{E}_{T V}\left(u_{j}\right)-C_{\mathrm{Per}}\left\|u_{j}\right\|_{p}^{p} \\
& \geq \mathcal{E}_{T V}\left(u_{j}\right)-C_{\mathrm{Per}}\left(C_{\mathrm{BV}}^{(p-1) n}\left\|u_{j}\right\|_{1}^{n-(n-1) p} \mathcal{E}_{T V}\left(u_{j}\right)+C_{\mathrm{BV}}^{(p-1) n}\left\|u_{j}\right\|_{1}^{n-(n-1) p}\right) \\
& =\mathcal{E}_{T V}\left(u_{j}\right)-C_{\mathrm{Per}} C_{\mathrm{BV}}^{(p-1) n} \alpha^{n-(n-1) p} \mathcal{E}_{T V}\left(u_{j}\right)-C_{\mathrm{Per}} C_{\mathrm{BV}}^{(p-1) n} \alpha^{n-(n-1) p} \\
& =\mathcal{E}_{T V}\left(u_{j}\right)\left(1-C_{\mathrm{Per}} C_{\mathrm{BV}}^{(p-1) n} \alpha^{n-(n-1) p}\right)-C_{\mathrm{Per}} C_{\mathrm{BV}}^{(p-1) n} \alpha^{n-(n-1) p}
\end{aligned}
$$

By (3.9) we obtain

$$
\mathcal{E}_{T V}\left(u_{j}\right) \leq \frac{c+1+C_{\mathrm{Per}} C_{\mathrm{BV}}^{(p-1) n} \alpha^{n-(n-1) p}}{1-C_{\mathrm{Per}} C_{\mathrm{BV}}^{(p-1) n} \alpha^{n-(n-1) p}}
$$

Whence, as above, $\left(u_{j}\right)_{j}$ is bounded in $B V(\Omega)$ and there is a convergent subsequence in $L^{p}(\Omega)$.

Proof of Theorem 3.5. We will apply Theorem 4.1 to

$$
\mathcal{F}=\mathcal{E}_{T V}+\mathcal{E}_{\mathrm{Per}}+I_{\left\{\mathcal{G}_{1}=\alpha\right\}}
$$

Obviously, (F1) is satisfied. (F2) is clearly satisfied in the case (E5') where $\mathcal{E}_{\text {Per }}$ is bounded from below. In the case (E5") we have $p \leq 1+\frac{1}{n}$ and, similar to (5.16), we use (E3), (4.5), and (3.9) to derive for $v \in B V(\Omega)$ with $\mathcal{G}_{1}(v)=\alpha$ that

$$
\begin{aligned}
\mathcal{F}(v) & =\mathcal{E}_{T V}(v)+\mathcal{E}_{\mathrm{Per}}(v) \\
& \geq \mathcal{E}_{T V}(v)-C_{\mathrm{Per}}\|v\|_{p}^{p} \\
& \geq\left(1-C_{\mathrm{Per}} C_{\mathrm{BV}}^{(p-1) n} \alpha^{n-(n-1) p}\right) \mathcal{E}_{T V}(v)-C_{\mathrm{Per}} C_{\mathrm{BV}}^{(p-1) n} \alpha^{n-(n-1) p} \\
& \geq-C_{\mathrm{Per}} C_{\mathrm{BV}}^{(p-1) n} \alpha^{n-(n-1) p} .
\end{aligned}
$$

Hence $\mathcal{F}$ is bounded from below and we have (F2) also in the second case. The 
function $\mathcal{F}$ satisfies the (PS)-condition by Proposition 3.3. The (epi)-condition follows from (5.15) (cf. [8, Thm. 3.4]). In order to verify (F5) we choose linearly independent $v_{1}, \ldots v_{k} \in C_{c}^{\infty}(\Omega)$ and a desired map $\Phi: \mathbb{S}^{k-1} \rightarrow L^{p}(\Omega)$ is obviously given by

$$
\Phi(x)=\Phi\left(x_{1}, \ldots, x_{k}\right)=\frac{\alpha \sum_{j=1}^{n} x_{j} v_{j}}{\left\|\sum_{j=1}^{n} x_{j} v_{j}\right\|_{1}} .
$$

The existence of a sequence of eigensolutions now follows from Theorem 4.1.

For the unboundedness of the critical values $\left(c_{k, \alpha}\right)_{k}$ we still need the compactness of the sublevel sets $\{\mathcal{F} \leq \gamma\}$. In the case of (E5') there is some $\tilde{\gamma} \in \mathbb{R}$ with $\mathcal{E}_{\text {Per }}(v) \geq \tilde{\gamma}$ for all $v \in L^{p}(\Omega)$. Hence

$$
0 \leq \mathcal{E}_{T V}(v)=\mathcal{F}(v)-\mathcal{E}_{\text {Per }}(v) \leq \gamma-\tilde{\gamma} \quad \text { for all } v \in\{\mathcal{F} \leq \gamma\}
$$

Since $\mathcal{E}_{T V}$ is an equivalent norm on $B V(\Omega)$, the set $\{\mathcal{F} \leq \gamma\}$ is bounded in $B V(\Omega)$ and, by the compact embedding $B V(\Omega) \hookrightarrow L^{p}(\Omega)$, it is compact in $L^{p}(\Omega)$. For the second case (E5") we argue analogously using (5.17) and (3.9).

For the assertion concerning the Euler-Lagrange equation we can obviously apply Theorem 3.1. It remains to show the boundedness statement for the rescaled family $v_{k, \alpha}=\frac{u_{k, \alpha}}{\left\|u_{k, \alpha}\right\|_{1}}$. This part of the proof is postponed to the end of this section.

Proof of Theorem 3.6. Using (3.3), (3.10), (4.5), $\mathscr{S}_{k}^{\alpha}$ according to (3.11), and

$$
\hat{c}_{k, \alpha}:=\frac{c_{k, \alpha}}{\alpha},
$$

we have

$$
\begin{aligned}
\limsup _{\alpha \rightarrow 0} \hat{c}_{k, \alpha} & =\limsup _{\alpha \rightarrow 0} \inf _{S \in \mathscr{S}_{k}^{\alpha}} \sup _{u \in S} \frac{1}{\alpha}\left(\mathcal{E}_{T V}(u)+\mathcal{E}_{\mathrm{Per}}(u)\right) \\
& =\limsup _{\alpha \rightarrow 0} \inf _{S \in \mathscr{S}_{k}^{1}} \sup _{u \in S}\left(\mathcal{E}_{T V}(u)+\frac{1}{\alpha} \mathcal{E}_{\mathrm{Per}}(\alpha u)\right) \\
& \leq \limsup _{\alpha \rightarrow 0} \inf _{S \in \mathscr{S}_{k}^{1}} \sup _{u \in S}\left(\mathcal{E}_{T V}(u)+\frac{1}{\alpha} C_{\mathrm{Per}}\|\alpha u\|_{p}^{p}\right) \\
& =\limsup _{\alpha \rightarrow 0} \inf _{S \in \mathscr{S}_{k}^{1}} \sup _{u \in S}\left(\mathcal{E}_{T V}(u)+\alpha^{p-1} C_{\mathrm{Per}}\|u\|_{p}^{p}\right) \\
& \leq \limsup _{\alpha \rightarrow 0} \inf _{S \in \mathscr{S}_{k}^{1}} \sup _{u \in S} \mathcal{E}_{T V}(u)\left(1+\alpha^{p-1} C_{\mathrm{BV}}^{(p-1) n} C_{\mathrm{Per}}\right)+\alpha^{p-1} C_{\mathrm{BV}}^{(p-1) n} C_{\mathrm{Per}} \\
& =\inf _{S \in \mathscr{S}_{k}^{1}} \sup _{u \in S} \mathcal{E}_{T V}(u)=\lambda_{k, 1} .
\end{aligned}
$$


Similarly, we obtain the reverse inequality by

$$
\begin{aligned}
\liminf _{\alpha \rightarrow 0} \hat{c}_{k, \alpha} & =\liminf _{\alpha \rightarrow 0} \inf _{S \in \mathscr{S}_{k}^{1}} \sup _{u \in S}\left(\mathcal{E}_{T V}(u)+\frac{1}{\alpha} \mathcal{E}_{\mathrm{Per}}(\alpha u)\right) \\
& \geq \limsup _{\alpha \rightarrow 0} \inf _{S \in \mathscr{S}_{k}^{1}} \sup _{u \in S}\left(\mathcal{E}_{T V}(u)-\frac{1}{\alpha} C_{\mathrm{Per}}\|\alpha u\|_{p}^{p}\right) \\
& \geq \limsup _{\alpha \rightarrow 0} \inf _{S \in \mathscr{S}_{k}^{1}} \sup _{u \in S} \mathcal{E}_{T V}(u)\left(1-\alpha^{p-1} C_{\mathrm{BV}}^{(p-1) n} C_{\mathrm{Per}}\right)-\alpha^{p-1} C_{\mathrm{BV}}^{(p-1) n} C_{\mathrm{Per}} \\
& =\inf _{S \in \mathscr{S}_{k}^{1}} \sup _{u \in S} \mathcal{E}_{T V}(u)=\lambda_{k, 1} .
\end{aligned}
$$

Hence

$$
\lim _{\alpha \rightarrow 0} \frac{c_{k, \alpha}}{\alpha}=\lim _{\alpha \rightarrow 0} \hat{c}_{k, \alpha}=\lambda_{k, 1}
$$

i.e. the fist assertion is verified. The other limit will follow from the next proposition.

Proposition 5.1. Let $\Omega \subseteq \mathbb{R}^{n}$ be open and bounded with Lipschitz boundary, let $\alpha>0$, let $1<p \leq 1+\frac{1}{n}$, and let $\mathcal{E}_{\text {Per }}$ satisfy (E1)-(E4). Moreover, $\left(u_{\alpha}\right)_{\alpha>0}$ be a family of critical points of (3.1), (3.2) with corresponding eigenvalues $\left(\lambda_{\alpha}\right)_{\alpha}$ such that the

$$
\hat{c}_{\alpha}:=\frac{1}{\alpha}\left(\mathcal{E}_{T V}\left(u_{\alpha}\right)+\mathcal{E}_{\mathrm{Per}}\left(u_{\alpha}\right)\right)
$$

are bounded for $0<\alpha \leq \alpha_{0}$ for some $\alpha_{0}>0$. Then the rescaled critical points

$$
v_{\alpha}:=\frac{u_{\alpha}}{\alpha}
$$

are bounded in $B V(\Omega)$ for $\alpha \in\left(0, \alpha_{1}\right]$ with some $\alpha_{1} \in\left(0, \alpha_{0}\right]$. Moreover,

$$
\left(\hat{c}_{\alpha}\right)_{\alpha} \text { converges as } \alpha \rightarrow 0 \text { if and only if }\left(\lambda_{\alpha}\right)_{\alpha} \text { converges as } \alpha \rightarrow 0
$$

and, in that case, we have

$$
\lim _{\alpha \rightarrow 0} \lambda_{\alpha}=\lim _{\alpha \rightarrow 0} \hat{c}_{\alpha}
$$

Proof. We use (3.3), (4.5), and $\left\|u_{\alpha}\right\|_{1}=\alpha$ to estimate

$$
\begin{aligned}
\hat{c}_{\alpha} & =\frac{1}{\alpha}\left(\mathcal{E}_{T V}\left(u_{\alpha}\right)+\mathcal{E}_{\mathrm{Per}}\left(u_{\alpha}\right)\right) \\
& \geq \mathcal{E}_{T V}\left(v_{\alpha}\right)-\frac{1}{\alpha} C_{\mathrm{Per}}\left\|\alpha v_{\alpha}\right\|_{p}^{p} \\
& =\mathcal{E}_{T V}\left(v_{\alpha}\right)-\alpha^{p-1} C_{\mathrm{Per}}\left\|v_{\alpha}\right\|_{p}^{p} \\
& \geq \mathcal{E}_{T V}\left(v_{\alpha}\right)-\alpha^{p-1} C_{\mathrm{Per}}\left(C_{\mathrm{BV}}^{(p-1) n}\left\|v_{\alpha}\right\|_{1}^{n-(n-1) p} \mathcal{E}_{T V}\left(v_{\alpha}\right)+C_{\mathrm{BV}}^{(p-1) n}\left\|v_{\alpha}\right\|_{1}^{n-(n-1) p}\right) \\
& =\left(1-\alpha^{p-1} C_{\mathrm{Per}} C_{\mathrm{BV}}^{(p-1) n}\right) \mathcal{E}_{T V}\left(v_{\alpha}\right)-\alpha^{p-1} C_{\mathrm{Per}} C_{\mathrm{BV}}^{(p-1) n} .
\end{aligned}
$$


For $\alpha \in\left(0, \alpha_{0}\right]$ sufficiently small, say $\alpha \leq \alpha_{1}$, we obtain

$$
\mathcal{E}_{T V}\left(v_{\alpha}\right) \leq \frac{\hat{c}_{\alpha}+\alpha^{p-1} C_{\mathrm{Per}} C_{\mathrm{BV}}^{(p-1) n}}{1-\alpha^{p-1} C_{\mathrm{Per}} C_{\mathrm{BV}}^{(p-1) n}} \leq \frac{\hat{c}_{\alpha}+\alpha_{1}^{p-1} C_{\mathrm{Per}} C_{\mathrm{BV}}^{(p-1) n}}{1-\alpha_{1}^{p-1} C_{\mathrm{Per}} C_{\mathrm{BV}}^{(p-1) n}}
$$

Hence $\mathcal{E}_{T V}\left(v_{\alpha}\right)$ is bounded and, since $\mathcal{E}_{T V}$ is an equivalent norm on $B V(\Omega)$, the first assertion follows.

Analogously to (5.19) we get

$$
\mathcal{E}_{T V}\left(v_{\alpha}\right) \geq \frac{\hat{c}_{\alpha}-\alpha^{p-1} C_{\mathrm{Per}} C_{\mathrm{BV}}^{(p-1) n}}{1+\alpha^{p-1} C_{\mathrm{Per}} C_{\mathrm{BV}}^{(p-1) n}} .
$$

By (3.8) there is some $u_{\alpha}^{*} \in \partial \mathcal{E}_{\text {Per }}\left(u_{\alpha}\right)$ with

$$
\lambda_{\alpha}=\frac{\mathcal{E}_{T V}\left(u_{\alpha}\right)+\left\langle u_{\alpha}^{*}, u_{\alpha}\right\rangle_{L^{p^{\prime}, L^{p}}}}{\alpha} .
$$

Using (3.4), (4.5), and (5.19) we can derive that

$$
\begin{aligned}
\lambda_{\alpha} & =\mathcal{E}_{T V}\left(\frac{u_{\alpha}}{\alpha}\right)+\alpha^{-1}\left\langle u_{\alpha}^{*}, u_{\alpha}\right\rangle_{L^{p^{\prime}, L^{p}}} \\
& \leq \mathcal{E}_{T V}\left(\frac{u_{\alpha}}{\alpha}\right)+\alpha^{-1} p C_{\mathrm{Per}}\left\|u_{\alpha}\right\|_{p}^{p} \\
& =\mathcal{E}_{T V}\left(v_{\alpha}\right)+\alpha^{p-1} p C_{\mathrm{Per}}\left\|v_{\alpha}\right\|_{p}^{p} \\
& \leq\left(1+\alpha^{p-1} p C_{\mathrm{Per}} C_{\mathrm{BV}}^{(p-1) n}\right) \mathcal{E}_{T V}\left(v_{\alpha}\right)+\alpha^{p-1} p C_{\mathrm{Per}} C_{\mathrm{BV}}^{(p-1) n} \\
& \leq\left(1+\alpha^{p-1} p C_{\mathrm{Per}} C_{\mathrm{BV}}^{(p-1) n}\right) \frac{\hat{c}_{\alpha}+\alpha^{p-1} C_{\mathrm{Per}} C_{\mathrm{BV}}^{(p-1) n}}{1-\alpha^{p-1} C_{\mathrm{Per}} C_{\mathrm{BV}}^{(p-1) n}}+\alpha^{p-1} p C_{\mathrm{Per}} C_{\mathrm{BV}}^{(p-1) n}
\end{aligned}
$$

Consequently,

$$
\limsup _{\alpha \rightarrow 0} \lambda_{\alpha} \leq \liminf _{\alpha \rightarrow 0} \hat{c}_{\alpha}
$$

With (5.20) we similarly obtain the opposite direction

$$
\begin{aligned}
\lambda_{\alpha} & =\mathcal{E}_{T V}\left(\frac{u_{\alpha}}{\alpha}\right)+\alpha^{-1}\left\langle u_{\alpha}^{*}, u_{\alpha}\right\rangle_{L^{p^{\prime}, L^{p}}} \\
& \geq \mathcal{E}_{T V}\left(\frac{u_{\alpha}}{\alpha}\right)-\alpha^{-1} p C_{\mathrm{Per}}\left\|u_{\alpha}\right\|_{p}^{p} \\
& \geq\left(1-\alpha^{p-1} p C_{\mathrm{Per}} C_{\mathrm{BV}}^{(p-1) n}\right) \frac{\hat{c}_{\alpha}-\alpha^{p-1} C_{\mathrm{Per}} C_{\mathrm{BV}}^{(p-1) n}}{1+\alpha^{p-1} C_{\mathrm{Per}} C_{\mathrm{BV}}^{(p-1) n}}-\alpha^{p-1} p C_{\mathrm{Per}} C_{\mathrm{BV}}^{(p-1) n}
\end{aligned}
$$


and, thus,

$$
\limsup _{\alpha \rightarrow 0} \hat{c}_{\alpha} \leq \liminf _{\alpha \rightarrow 0} \lambda_{\alpha}
$$

Now the assertion follows from (5.21) and (5.22).

We continue with the proof of Theorem 3.6 by applying Proposition 5.1 to $\left(u_{k, \alpha}\right)_{\alpha}$ and $\left(\lambda_{k, \alpha}\right)_{\alpha}$. Using (5.18) we conclude that

$$
\lim _{\alpha \rightarrow 0} \lambda_{k, \alpha}=\lim _{\alpha \rightarrow 0} \hat{c}_{k, \alpha}=\lambda_{k, 1}
$$

which completes the proof.

Proof of Theorem [3.5. We still have to show that, for fixed $k \in \mathbb{N}$, there is some $\alpha_{1}>0$ such that the family $v_{k, \alpha}=\frac{u_{k, \alpha}}{\left\|u_{k, \alpha}\right\|_{1}}$ is bounded in $B V(\Omega)$ for $\alpha \in\left(0, \alpha_{1}\right]$. But, by (5.18), this is a direct consequence of Proposition 5.1 applied to $\left(u_{k, \alpha}\right)_{\alpha}$ and $\left(\lambda_{k, \alpha}\right)_{\alpha}$.

\subsection{Proofs for perturbations of the constraint}

Proof of Theorem 3.7. It is not difficult to see that we can apply [8, Cor. 3.7] with

$$
f_{0}=\mathcal{E}_{T V}, f_{1}=0, g_{0}=-1, g_{1}=\mathcal{G}_{1}+\mathcal{G}_{\mathrm{Per}}-\beta .
$$

As in the proof of Theorem 3.1, the (epi)-condition follows from [8, Thm. 3.4]) with $u_{-}=0$ and $u_{+}=2 u$ by the preceding lemma.

Let us now prepare the proof of Theorem 3.9.

Lemma 5.2. Let $\mathcal{G}_{\text {Per }}$ be locally Lipschitz continuous such that (G5) is satisfied and let $u \in L^{p}(\Omega) \backslash\{0\}$. Then the function

$$
t \mapsto \mathcal{G}_{1}(t u)+\mathcal{G}_{\mathrm{Per}}(t u)
$$

is strictly increasing on $[0, \infty)$ and we have

$$
\left(\mathcal{G}_{1}+\mathcal{G}_{\mathrm{Per}}\right)^{0}(u ;-u)<0
$$

Proof. Let $\mathcal{G}:=\mathcal{G}_{1}+\mathcal{G}_{\text {Per }}$ and let $0 \leq t_{1}<t_{2}$. By Lebourg's Theorem (cf. [3, Thm. 2.3.7]) there is $\theta \in(0,1)$ and $w^{*} \in \partial \mathcal{G}\left(\left(\theta t_{1}+(1-\theta) t_{2}\right) u\right)$ such that

$$
\mathcal{G}\left(t_{2} u\right)-\mathcal{G}\left(t_{1} u\right)=\left\langle w^{*},\left(t_{2}-t_{1}\right) u\right\rangle=\left(t_{2}-t_{1}\right) \int_{\Omega} w^{*}(x) u(x) \mathrm{d} x .
$$


By the sum rule for generalized gradients (cf. [3, Prop. 2.3.3]) we find $s \in \partial \mathcal{G}_{1}(u)$ and $u^{*} \in \mathcal{G}_{\mathrm{Per}}(u)$ with $w^{*}=s+u^{*}$ where $s(x) \in \operatorname{Sgn}(u(x))$ for a.e. $x \in \Omega$ (cf. [13]). Whence we have for almost every $x \in \Omega$ with $u(x) \neq 0$ that

$$
w^{*}(x) u(x)=s(x) u(x)+u^{*}(x) u(x)=|u(x)|+u^{*}(x) u(x)>0
$$

by (3.17). With (5.24) we obtain the first assertion that $t \mapsto \mathcal{G}(t u)$ is strictly increasing.

Using [3, Prop. 2.1.2] and (3.17) we get for the generalized directional derivative

$$
\begin{aligned}
\mathcal{G}^{0}(u ;-u) & =\max _{w^{*} \in \partial \mathcal{G}(u)}\left\langle w^{*},-u\right\rangle \\
& \leq \max _{s \in \partial \mathcal{G}_{1}(u)}\langle s,-u\rangle+\max _{u^{*} \in \partial \mathcal{G}_{\operatorname{Per}}(u)}\left\langle u^{*},-u\right\rangle \\
& =-\int_{\Omega}|u(x)| \mathrm{d} x+\max _{u^{*} \in \partial \mathcal{G}_{\operatorname{Per}}(u)}-\int_{\Omega} u^{*}(x) u(x) \mathrm{d} x \\
& =-\|u\|_{1}-\min _{u^{*} \in \partial \mathcal{G}_{\operatorname{Per}}(u)} \int_{\Omega} u^{*}(x) u(x) \mathrm{d} x \\
& <-\|u\|_{1}-\int_{\Omega}-|u(x)| \mathrm{d} x \\
& =-\|u\|_{1}+\|u\|_{1}=0
\end{aligned}
$$

and the proof is complete.

Lemma 5.3. We assume that the assumptions of Theorem 3.9 are satisfied and that $u \in L^{p}(\Omega) \backslash\{0\}$ is given. Then there exists a unique $t_{u}>0$ such that

$$
\mathcal{G}_{1}\left(t_{u} u\right)+\mathcal{G}_{\mathrm{Per}}\left(t_{u} u\right)=\beta
$$

The mapping $u \mapsto t_{u}$ is continuous and even on $L^{p}(\Omega) \backslash\{0\}$. Moreover

$$
\Phi_{\beta}:\left\{u \in L^{p}(\Omega) ; \mathcal{G}_{1}(u)+\mathcal{G}_{\mathrm{Per}}(u)=\beta\right\} \rightarrow\left\{u \in L^{p}(\Omega) ;\|u\|_{1}=1\right\}
$$

given by

$$
\Phi_{\beta}(u)=\frac{u}{\|u\|_{1}}
$$

is an odd homeomorphism with odd inverse $\Psi_{\beta}$ given by

$$
\Psi_{\beta}(u)=t_{u} u \quad \text { for } u \in L^{p}(\Omega) \text { with }\|u\|_{1}=1 \text {. }
$$


Proof. By Lemma 5.2 the mapping

$$
[0, \infty) \ni t \mapsto\|t u\|_{1}+\mathcal{G}_{\mathrm{Per}}(t u)
$$

is strictly increasing and, obviously, it is continuous. From assumption (3.14) we infer

$$
\|0 u\|_{1}+\mathcal{G}_{\mathrm{Per}}(0 u)=0 \quad \text { and } \quad \liminf _{t \rightarrow \infty}\|t u\|_{1}+\mathcal{G}_{\mathrm{Per}}(t u) \geq \liminf _{t \rightarrow \infty} t\|u\|_{1}=\infty
$$

Thus $t_{u}$ exists by the intermediate value theorem and is uniquely determined by strict monotonicity. Since $\mathcal{G}_{1}$ and $\mathcal{G}_{\text {Per }}$ are even, also $u \mapsto t_{u}$ is even.

Let now $u_{j} \rightarrow u \neq 0$ in $L^{p}\left(\Omega\right.$ and, thus, also in $L^{1}(\Omega)$. With $t_{j}:=t_{u_{j}}$ and $\mathcal{G}_{\text {Per }}\left(u_{j}\right) \geq 0$ by (3.14) we have $\beta \geq t_{j}\left\|u_{j}\right\|_{1}$. Therefore $\left(t_{j}\right)_{j}$ must be bounded and, at least for a subsequence (denoted the same way), we get $t_{j} \rightarrow: t_{0} \geq 0$. By continuity

$$
\beta=\lim _{j \rightarrow \infty} \mathcal{G}_{1}\left(t_{j} u_{j}\right)+\mathcal{G}_{\mathrm{Per}}\left(t_{j} u_{j}\right)=\left\|t_{0} u\right\|_{1}+\mathcal{G}_{\mathrm{Per}}\left(t_{0} u\right)
$$

Uniqueness of $t_{u}$ implies $t_{0}=t_{u}$ and, thus, continuity of $u \mapsto t_{u}$. The properties of $\Phi_{\beta}$ and $\Psi_{\beta}$ are a simple consequence of the properties of $t_{u}$.

Proof of Theorem 3.9. We will apply Theorem 4.1 to $\mathcal{F}=\mathcal{E}_{T V}+I_{\left\{\mathcal{G}_{1}+\mathcal{G}_{\text {Per }}=\beta\right\}}$. Properties (F1) and (F2) are immediate. Since $\mathcal{E}_{T V}$ is an equivalent norm on $B V(\Omega)$, the sublevel sets $\{\mathcal{F} \leq c\}$ are obviously bounded in $B V(\Omega)$ and, by the compact embedding $B V(\Omega) \hookrightarrow L^{p}(\Omega)$, they are compact in $L^{p}(\Omega)$. Clearly, any (PS)-sequence for the level $c \in \mathbb{R}$ is eventually contained in $\{\mathcal{F} \leq c+1\}$ and, therefore, compactness of all sublevel sets implies the (PS)-condition. The (epi)-condition follows from (5.23) and [8, Thm. 3.4] applied with

$$
g_{0}=-1, g_{1}=\mathcal{G}_{1}+\mathcal{G}_{\mathrm{Per}}-\beta, u_{-}=0, u_{+}=2 u, C=B V(\Omega)
$$

Using $\Phi: \mathbb{S}^{k-1} \rightarrow\left\{\|\cdot\|_{1}=1\right\} \subseteq L^{p}(\Omega)$ from the proof of Theorem 3.5 with $\alpha=1$, the mapping $\Psi_{\beta} \circ \Phi: \mathbb{S}^{k-1} \rightarrow\left\{\mathcal{G}_{1}+\mathcal{G}_{\text {Per }}=\beta\right\} \subseteq L^{p}(\Omega)$ verifies assumption (F5). Now Theorem 4.1 implies the stated existence of a sequence of eigensolutions of (3.12), (3.13) and the unboundedness of the sequence of critical values $\left(c_{k, \beta}\right)_{k}$. Clearly we can apply Theorem 3.7 for the assertion concerning the Euler-Lagrange equation.

It remains to show that, for fixed $k \in \mathbb{N}$, the rescaled family $v_{k, \beta}=\frac{u_{k, \beta}}{\left\|u_{k, \beta}\right\|_{1}}$ is bounded in $B V(\Omega)$ for $\beta>0$ bounded. We postpone this part of the proof to the end of this section. 
Proof of Theorem 3.10. With condition (3.14) and Corollary 4.4, we have for any $u \in L^{p}(\Omega) \cap B V(\Omega)$ with $\mathcal{G}_{1}(u)+\mathcal{G}_{\text {Per }}(u)=\beta$ that

$$
\begin{aligned}
\|u\|_{1} \leq \beta & =\|u\|_{1}+\mathcal{G}_{\mathrm{Per}}(u) \\
& \leq\|u\|_{1}+C_{\mathrm{Per}}\|u\|_{p}^{p} \\
& \leq\|u\|_{1}+C_{\mathrm{Per}} C_{\mathrm{BV}}^{(p-1) n}\|u\|_{1}^{n-(n-1) p} \mathcal{E}_{T V}(u)^{(p-1) n} \\
& =\|u\|_{1}\left(1+C_{\mathrm{Per}} C_{\mathrm{BV}}^{(p-1) n}\|u\|_{1}^{p-1} \mathcal{E}_{T V}\left(\frac{u}{\|u\|_{1}}\right)^{(p-1) n}\right) \\
& \leq\|u\|_{1}\left(1+C_{\mathrm{Per}} C_{\mathrm{BV}}^{(p-1) n} \beta^{p-1} \mathcal{E}_{T V}\left(\frac{u}{\|u\|_{1}}\right)^{(p-1) n}\right) .
\end{aligned}
$$

Consider $\Phi_{\beta}$ from Lemma 5.3 and $\mathscr{S}_{k}^{1}$ according to (3.11) with $\alpha=1$. Since the genus remains unchanged under homeomorphisms, we have

$$
S \in \mathscr{S}_{k}^{\beta} \quad \text { if and only if } \quad \Phi_{\beta}(S) \in \mathscr{S}_{k}^{1} .
$$

Therefore

$$
\begin{aligned}
\hat{c}_{k, \beta}:=\frac{c_{k, \beta}}{\beta} & =\inf _{S \in \mathscr{S}_{k}^{\beta}} \sup _{u \in S} \frac{1}{\beta} \mathcal{E}_{T V}(u) \\
& =\inf _{S \in \mathscr{S}_{k}^{\beta}} \sup _{u \in S} \frac{\|u\|_{1}}{\beta} \mathcal{E}_{T V}\left(\frac{u}{\|u\|_{1}}\right) \\
& \leq \inf _{S \in \mathscr{S}_{k}^{\beta}} \sup _{u \in S} \frac{\beta}{\beta} \mathcal{E}_{T V}\left(\frac{u}{\|u\|_{1}}\right) \\
& =\inf _{S \in \mathscr{S}_{k}^{1}} \sup _{v \in S} \mathcal{E}_{T V}(v)=\lambda_{k, 1} .
\end{aligned}
$$

For some reverse inequality we choose $S_{\beta} \in \mathscr{S}_{k}^{\beta}$ with

$$
\hat{c}_{k, \beta}=\frac{c_{k, \beta}}{\beta}=\sup _{u \in S_{\beta}} \frac{\mathcal{E}_{T V}(u)}{\beta}
$$

for any $\beta>0$ according to Theorem 4.1. By (5.30) and (5.27) we have for $u \in S_{\beta}$

$$
\begin{aligned}
\lambda_{k, 1} \geq \hat{c}_{k, \beta} & =\sup _{u \in S_{\beta}} \frac{\mathcal{E}_{T V}(u)}{\beta} \\
& \geq \sup _{u \in S_{\beta}} \frac{\mathcal{E}_{T V}(u)}{\|u\|_{1}\left(1+C_{\mathrm{Per}} C_{B V}^{(p-1) n}\|u\|_{1}^{p-1} \mathcal{E}_{T V}\left(\frac{u}{\|u\|_{1}}\right)^{(p-1) n}\right)} .
\end{aligned}
$$


Consequently,

$$
\lambda_{k, 1}\left(1+C_{\mathrm{Per}} C_{B V}^{(p-1) n} \beta^{p-1} \mathcal{E}_{T V}\left(\frac{u}{\|u\|_{1}}\right)^{(p-1) n}\right) \geq \mathcal{E}_{T V}\left(\frac{u}{\|u\|_{1}}\right) \quad \text { for } u \in \bigcup_{\beta>0} S_{\beta} .
$$

The above inequality is of linear growth in $\mathcal{E}\left(\frac{u}{\|u\|_{1}}\right)$ on the right had side and, by $p<1+\frac{1}{n}$, of sublinear growth on the left hand side. Thus, for any $\beta_{0}>0$ there is some $\tilde{C}>0$ such that

$$
\mathcal{E}_{T V}\left(\frac{u}{\|u\|_{1}}\right) \leq \tilde{C} \quad \text { for } u \in S_{\beta}, 0<\beta \leq \beta_{0} .
$$

By (5.28), (5.29), and the definition of $\lambda_{k, 1}$ in (1.11), we now find some $C>0$ with

$$
\begin{aligned}
\hat{c}_{k, \beta}=\sup _{u \in S_{\beta}} \frac{\mathcal{E}_{T V}(u)}{\beta} & \geq \frac{1}{1+C \beta^{p-1}} \sup _{u \in S_{\beta}} \mathcal{E}_{T V}\left(\frac{u}{\|u\|_{1}}\right) \\
& \geq \frac{\lambda_{k, 1}}{1+C \beta^{p-1}} \quad \text { for } \beta \leq \beta_{0} .
\end{aligned}
$$

Using (5.30) we readily derive the first assertion that

$$
\lim _{\beta \rightarrow 0} \frac{c_{k, \beta}}{\beta}=\lim _{\beta \rightarrow 0} \hat{c}_{k, \beta}=\lambda_{k, 1}
$$

For the other limit in (3.23) we first recall that $c_{k, \beta}=\mathcal{E}_{T V}\left(u_{k, \beta}\right)$. Testing the Euler-Lagrange Equation (3.20) with $u_{k, \beta}$, we obtain

$$
\hat{c}_{k, \beta}=\frac{\mathcal{E}_{T V}\left(u_{k, \beta}\right)}{\beta}=\lambda_{k, \beta} \frac{\left\|u_{k, \beta}\right\|_{1}+\left\langle u_{k, \beta}^{*}, u_{k, \beta}\right\rangle}{\beta}
$$

for some $u_{k, \beta}^{*} \in \partial \mathcal{G}_{\mathrm{Per}}\left(u_{k, \beta}\right)$. Thus the remaining result follows if we show that

$$
\lim _{\beta \rightarrow 0} \frac{\left\|u_{k, \beta}\right\|_{1}+\left\langle u_{k, \beta}^{*}, u_{k, \beta}\right\rangle}{\beta}=1
$$

By the continuous embedding $B V(\Omega) \hookrightarrow L^{p}(\Omega)$ and since $\mathcal{E}_{T V}$ is an equivalent norm on $B V(\Omega)$, there is some $C>0$ such that

$$
\|v\|_{p} \leq C \mathcal{E}_{T V}(v) \text { for } v \in B V(\Omega) .
$$

Using (3.15) we get for some possibly larger $C>0$

$$
\left|\left\langle u_{k, \beta}^{*}, u_{k, \beta}\right\rangle\right| \leq p C_{\mathrm{Per}}\left\|u_{k, \beta}\right\|_{p}^{p} \leq C \mathcal{E}_{T V}\left(u_{k, \beta}\right)^{p}
$$


Thus, with (5.25) and (5.33),

$$
\limsup _{\beta \rightarrow 0} \frac{\left\|u_{k, \beta}\right\|_{1}+\left\langle u_{k, \beta}^{*}, u_{k, \beta}\right\rangle}{\beta} \leq \limsup _{\beta \rightarrow 0} \frac{\beta+C c_{k, \beta}^{p}}{\beta}=1 .
$$

Since $\left\|u_{k, \beta}\right\|_{1} \geq \beta-C_{\mathrm{Per}}\left\|u_{k, \beta}\right\|_{p}^{p}$ by (15.26), we find some $\hat{C}>0$ with

$$
\liminf _{\beta \rightarrow 0} \frac{\left\|u_{k, \beta}\right\|_{1}+\left\langle u_{k, \beta}^{*}, u_{k, \beta}\right\rangle}{\beta} \geq \liminf _{\beta \rightarrow 0} \frac{\beta-\hat{C} c_{k, \beta}^{p}}{\beta}=1 .
$$

But this verifies (5.35) and the proof is complete.

Proof of Theorem [3.9, second part. Let $k \in \mathbb{N}$ and any $\beta_{0}>0$ be fixed. For (arbitrary) critical points $u_{k, \beta}$ of (3.12), (3.13) with critical value $c_{k}$ it remains to show that the family $v_{k, \beta}=\frac{u_{k, \beta}}{\left\|u_{k, \beta}\right\|_{1}}$ is bounded in $B V(\Omega)$ for $0<\beta<\beta_{0}$. By (5.30) and (5.28) we obtain

$$
\lambda_{k, 1} \geq \frac{c_{k, \beta}}{\beta}=\frac{\mathcal{E}_{T V}\left(u_{k, \beta}\right)}{\beta} \geq \frac{\mathcal{E}_{T V}\left(u_{k, \beta}\right)}{\left\|u_{k, \beta}\right\|_{1}\left(1+C_{\mathrm{Per}} C_{\mathrm{BV}}^{(p-1) n} \beta^{p-1} \mathcal{E}_{T V}\left(\frac{u_{k, \beta}}{\left\|u_{k, \beta}\right\|_{1}}\right)^{(p-1) n}\right)} .
$$

Consequently, for $0<\beta<\beta_{0}$,

$$
\lambda_{k, 1}\left(1+C_{\mathrm{Per}} C_{\mathrm{BV}}^{(p-1) n} \beta_{0}^{p-1} \mathcal{E}_{T V}\left(v_{k, \beta}\right)^{(p-1) n}\right) \geq \mathcal{E}_{T V}\left(v_{k, \beta}\right) .
$$

Analogously to the arguments giving (5.32), we use the sublinear and linear growth in $\mathcal{E}_{T V}\left(v_{k, \beta}\right)$ to derive a uniform bound on $\mathcal{E}_{T V}\left(v_{k, \beta}\right)$ for $0<\beta<\beta_{0}$. Since $\mathcal{E}_{T V}$ is an equivalent norm on $B V(\Omega)$, the assertion follows.

\section{References}

[1] L. Ambrosio, P. Tilli. Topics on Analysis in Metric Spaces. Oxford University Press, Oxford, 2004.

[2] I. Cioranescu. Geometry of Banach Spaces, Duality Mappings and Nonlinear Problems. Kluwer Academic Publishers, Dordrecht, 1990.

[3] F. Clarke. Optimization and Nonsmooth Analysis. Canadian Mathematical Society Series of Monographs and Advanced Texts, John Wiley \& Sons, New York, 1987.

[4] J.-N. Corvellec. A general approach to the min-max principle. Z. Anal. Anwend. 16 (1997) 405-433. 
[5] J.-N. Corvellec, M. Degiovanni, M. Marzocchi. Deformation properties for continuous functionals and critical point theory. Topol. Methods Nonlinear Anal. 1 (1993) 151-171.

[6] M. Degiovanni, P. Magrone. Linking solutions for quasilinear equations at critical growth involving the "1-Laplace" operator. Calc. Var. Partial Differential Equations 36 (2009) 591-609.

[7] M. Degiovanni, M. Marzocchi. A critical point theory for nonsmooth functionals. Ann. Mat. Pura Appl. (IV) 167 (1994) 73-100.

[8] M. Degiovanni, F. Schuricht. Multiplicity results for free and constrained nonlinear elastic rods based on nonsmooth critical point theory. Math. Ana. 331 (1998) 675-728.

[9] M. A. del Pino, R. F. Manásevich. Global bifurcation of the $p$-Laplacian. J. Differ. Equ. 92 (1991) 226-251.

[10] L. C. Evans, R. F. Gariepy. Measure Theory and Fine Properties of Functions (Studies in Advanced Mathematics). CRC-Press, Boca Raton, 1992.

[11] E. Fadell. The relationship between Ljusternik-Schnirelman category and the concept of genus. Pacific J. M. 89 (1980) 33-42.

[12] J. P. Garcia Azorero, I. Peral Alonso. Existence and nonuniqueness for the p-Laplacian. Comm. Partial Differential Equations 12 (1987) 1389-1430.

[13] B. Kawohl, F. Schuricht. Dirichlet problems for the 1-Laplace operator, including the eigenvalue problem. Commun. Contemp. Math. 9 (2007) 513543.

[14] S. Littig, F. Schuricht. Convergence of the eigenvalues of the $p$-Laplace operator as $p$ goes to 1. Calc. Var. Partial Differential Equations. 49 (2014) $707-727$.

[15] Z. Milbers, F. Schuricht. Existence of a sequence of eigensolutions for the 1-Laplace operator. J. Lond. Math. Soc. 82 (2010) 74-88.

[16] Z. Milbers, F. Schuricht. Some special aspects related to the 1-Laplace operator. Adv. Calc. Var. 4 (2010) 101-126.

[17] Z. Milbers, F. Schuricht. Necessary condition for eigensolutions of the 1Laplace operator by means of inner variations. Math. Ann. 356 (2013) 147-177. 
[18] E. Parini. Continuity of the variational eigenvalues of the $p$-Laplacian with respect to p. Bull. Aust. Math. Soc. 83 (2011) 376-381.

[19] I. Peral. Multiplicity of solutions for the $p$-Laplacian. Intern. Center for Theoretical Physics Trieste, Second School of Nonlinear Functional Analysis and Applications to Differential Equations, 21 April - 9 May 1997.

[20] P. Rabinowitz. Some aspects of nonlinear eigenvalue problems. Rocky Mountain J. Math. 3 (1973) 161-202.

[21] E. Zeidler. Nonlinear Functional Analysis and its Applications: Part 3: Variational Methods and Optimization. Springer, Berlin, 1984. 